\title{
Semiclassical Analysis with Vanishing Magnetic Fields
}

\author{
Nicolas Dombrowski and Nicolas Raymond
}

\begin{abstract}
We analyze the 2D magnetic Laplacian in the semiclassical limit in the case when the magnetic field vanishes along a smooth curve. In particular, we prove local and microlocal estimates for the eigenfunctions and a complete asymptotic expansion of the eigenpairs in powers of $h^{1 / 6}$.
\end{abstract}

Mathematics Subject Classification (2010). 35P15, 81Q20.

Keywords. Semi-classical limit, microlocal analysis, quasimode, Agmon estimates, BornOppenheimer approximation.

\section{Contents}

1 Introduction . . . . . . . . . . . . . . . . 423

2 Construction of quasimodes . . . . . . . . . . . . . . . 431

3 Local and microlocal estimates . . . . . . . . . . . . . . . . 437

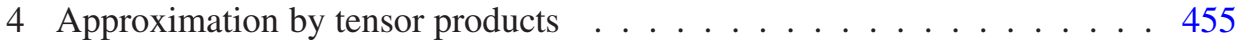

References . . . . . . . . . . . . . . . . . . . . . . 461

\section{Introduction}

We consider a vector potential $\mathbf{A} \in \mathcal{C}^{\infty}\left(\mathbb{R}^{2}, \mathbb{R}^{2}\right)$ and we consider the self-adjoint operator defined by:

$$
\mathscr{L}_{h, \mathbf{A}}=(-i h \nabla+\mathbf{A})^{2},
$$

where $h>0$ is the semiclassical parameter: We are interested in the limit $h \rightarrow 0$. This operator is gauge invariant. Indeed, for a smooth and real-valued function $\varphi$, we have

$$
e^{-i \varphi / h} \mathscr{L}_{h, \mathbf{A}} e^{i \varphi / h}=\mathscr{L}_{h, \mathbf{A}+\nabla \varphi}
$$

Therefore the spectrum of $\mathscr{L}_{h, \mathbf{A}}$ only depends on the magnetic field $\beta=\nabla \times \mathbf{A}$.

Notation 1.1. We will denote by $\lambda_{n}(h)$ the $n$-th eigenvalue of $\mathscr{L}_{h, \mathbf{A}}$. 
The aim of this paper is to give asymptotic expansions of $\lambda_{n}(h)$ when $h \rightarrow 0$. Let us notice that this regime is equivalent to the strong magnetic field limit which is often involved in applications (superconductivity, Hall regime etc.).

Framework and state of the art. There are essentially four motivations to the present analysis. The first one comes from the theory of superconductivity in which the magnetic Laplacian appears in the study of the third critical field associated with the Ginzburg-Landau functional (see [29], [30], and also the book [12] and the references therein).

The second one is related to the papers of R. Montgomery [32], X-B. Pan and K-H. Kwek [33] and B. Helffer and Y. Kordyukov [18] (see also [20] and [16]) where the authors analyze the spectrum of the magnetic Laplacian when the magnetic field vanishes along a smooth curve. In these papers, the main question is to know if the cancellation of the magnetic field can be seen on the semiclassical expansion of the eigenpairs (until now only the first term of the asymptotics is known for $\lambda_{n}(h)$, see [18], Corollary 1.1, when $k=1$ ). Coming from geometrical motivations, Montgomery was interested by the geometrical aspect of the magnetic field. More precisely, the magnetic Hamiltonian is the Laplacian associated to a connection whose curvature is the magnetic field (see [32] for more details). Our results complete these considerations in the sense that the asymptotics of [32] only gives the leading term whereas our method will give the complete structure of the spectrum in the semiclassical limit.

The third motivation appears in the recent paper [10] where the authors are concerned with the "magnetic waveguides". Among other questions they analyze the case of a magnetic barrier, that is of a piecewise constant magnetic field in $\mathbb{R}^{2}$. In particular they investigate the case when the field takes two opposite values and enlighten the classical "snake orbits" along the jump through the semiclassical limit. It turns out that the singularity (arising along a line) of the magnetic field in their paper seems to play the role of a vanishing magnetic field. The main application is related to new type of semi-conductor devices for which the transport caused by Quantum Hall-effect (QHE) would be played by such magnetic phenomenons. The important fact proved in [10] is that such phenomenons are intrinsic to the system (in the sense that the transport is topologically quantized). For the physical counterpart of these results one refers to [38] and [14].

The last motivation is to understand at which point there is an analogy between the magnetic Laplacian and the Laplacian with electric potential (see the papers [24], [25], and [15]). For instance, it is clear that if we translate the electric potential by a constant, then the spectrum is translated by a constant; but if we translate the magnetic field by a constant, we will see in this paper that the semiclassical analysis is strongly changed. Moreover this paper also aims at enlightening that, at some point, the magnetic Laplacian can be reduced to the electric Laplacian thanks to a local and microlocal analysis and unitary transforms (as it is the case when the magnetic field is 
positive in [11] and [37]). Part of our analysis will use the Feshbach-Grushin method (also called Lyapunov-Schmidt reduction and which is a resolvent approximation result) and a homogenization process involving multiple-scale expansions (see for instance the recent work [26] where the same philosophy appears in another context).

Let us now recall the nature of the known results concerning the asymptotic expansion of the eigenvalues of the magnetic Laplacian. When the magnetic field is constant in $2 \mathrm{D}$, there are many results concerning the two terms asymptotics of the lowest eigenvalue $\lambda_{1}(h)$ (see [2], [3], [9], and [21] in the case of a smooth and bounded domain with the Neumann condition on the boundary); the asymptotics at any order of all the lowest eigenvalues is proved in [11]. In the Neumann case, when the magnetic field is generically non constant and positive, the one term asymptotics is given in [29], the two terms asymptotics in [34] and at any order in [37]. For the Dirichlet case, the complete asymptotics is done in [19]. When the magnetic field cancels, the main results concern the one term asymptotics of the eigenvalues and the eigenfunctions concentration near the zero locus of the magnetic field (see [32] and [33]). In 3D, the two terms expansion is performed in [22] whereas in the variable case this problem is analyzed in [35] and [36].

General Assumptions on $\boldsymbol{\beta}$. Let us describe the main frame of this paper. In order $\mathscr{L}_{h, \mathbf{A}}$ to have compact resolvent, we will assume that

$$
\beta(x) \underset{|x| \rightarrow+\infty}{\longrightarrow}+\infty .
$$

As in [33] and [18], we will investigate the case when $\beta$ cancels along a closed and smooth curve $\Gamma$ in $\mathbb{R}^{2}$. Let us notice that the assumption (1.1) could clearly be relaxed so that one could also consider a smooth, bounded and simply connected domain of $\mathbb{R}^{2}$ with Dirichlet or Neumann condition on the boundary as far as the magnetic field does not vanish near the boundary. Nevertheless we do not strive for maximum generality the present "generic" case giving enough information when the magnetic field "nicely" cancels (one could also make it to cancel at a higher order as in [18]). We let

$$
\Gamma=\{\gamma(s), s \in \mathbb{R}\}
$$

We assume that $\beta$ is non positive inside $\Gamma$ and non negative outside. We introduce the standard tubular coordinates $(s, t)$ near $\Gamma$,

$$
\Phi(s, t)=\gamma(s)+t v(s)
$$

where $v(s)$ denotes the inward pointing normal to $\Gamma$ at $\gamma(s)$. We let:

$$
\tilde{\beta}(s, t)=\beta(\Phi(s, t))
$$

so that:

$$
\tilde{\beta}(s, 0)=0 .
$$


Heuristics and leading operator. Let us adopt first a heuristic point of view to introduce the leading operator of the analysis presented in this paper. We want to describe the operator $\mathscr{L}_{h, \mathbf{A}}$ near the cancellation line of $\beta$, that is near $\Gamma$. In a rough approximation, near $\left(s_{0}, 0\right)$, we can imagine that the line is straight $(t=0)$ and that the magnetic field cancels linearly so that we can consider $\tilde{\beta}(s, t)=\delta\left(s_{0}\right) t$ where $\delta\left(s_{0}\right)$ is the derivative of $\tilde{\beta}$ with respect to $t$. Therefore the operator to which it seems we are reduced at the leading order near $s_{0}$ is

$$
h^{2} D_{t}^{2}+\left(h D_{s}-\delta\left(s_{0}\right) \frac{t^{2}}{2}\right)^{2}
$$

1.1. The Montgomery operator. As in [32], [33], and [18], we will be led to investigate the following operator (self-adjoint realization on $\mathbb{R}$ ) with parameters $\eta \in \mathbb{R}$ and $\delta>0$,

$$
H_{\eta, \delta}=D_{t}^{2}+\left(-\eta+\frac{\delta}{2} t^{2}\right)^{2}
$$

where we have used the notation:

Notation 1.2. If $y$ is a variable, we let $D_{y}=-i \partial_{y}$.

We can also refer to [22], Section 2.4, where this operator appears. In fact, this operator is sometimes called Montgomery operator (see [32]). This operator is a generic example of a larger class of anharmonic oscillators (see [23]). We will see that it will be involved in the asymptotics at the first order whereas the second order will be related to a harmonic oscillator.

The Montgomery operator has clearly compact resolvent and we can consider its lowest eigenvalue denoted by $v_{\delta}(\eta)$. In fact, $v_{\delta}$ is related to $v_{1}$. Indeed, we can perform a rescaling $t=\delta^{-1 / 3} \tau$ so that $H_{\eta, \delta}$ is unitarily equivalent to

$$
\delta^{2 / 3}\left(D_{\tau}^{2}+\left(-\eta \delta^{-1 / 3}+\frac{1}{2} \tau^{2}\right)^{2}\right)=\delta^{2 / 3} H_{\eta \delta^{-1 / 3}, 1} .
$$

It is known (see [17] and [23]) that, for all $\delta>0$,

$\eta \mapsto v_{\delta}(\eta)$ admits a unique and non-degenerate minimum at a point $\eta_{0}$.

We may write

$$
\inf _{\eta \in \mathbb{R}} v_{\delta}(\eta)=\delta^{2 / 3} v_{1}\left(\eta_{0}\right)
$$

Notation 1.3. We let $H_{\eta}=H_{\eta, 1}$ and we denote by $u_{\eta}$ the $L^{2}$-normalized and positive eigenfunction associated with $v_{1}(\eta)$.

For fixed $\delta>0$, the family $\left(H_{\eta, \delta}\right)_{\eta \in \mathbb{R}}$ is an analytic family of type $(B)$ so that the eigenpair $\left(v_{1}(\eta), u_{\eta}\right)$ has an analytic dependence on $\eta$ (see [28]). 
Numerical computations of the $\eta_{0}$ and $\nu_{\eta_{0}}$ are performed by V. Bonnaillie Noël (see [23], Table 1) and give $\eta_{0} \approx 0.35$ and $\nu_{1}\left(\eta_{0}\right) \approx 0,57$. It is also proved that

$$
\lim |\eta| \rightarrow+\infty v_{1}(\eta)=+\infty
$$

Feynman-Hellmann Theorem. Let us recall a few formulas justified by the perturbation theory of Kato and known as "Feynman-Hellmann" formulas.

Lemma 1.4. We have

$$
\left(H_{\eta_{0}}-v\left(\eta_{0}\right)\right) v_{\eta_{0}}=-\left.\left(\partial_{\eta} H_{\eta}\right)\right|_{\eta=\eta_{0}} u_{\eta_{0}}
$$

with $v_{\eta_{0}}=\left.\left(\partial_{\eta} u_{\eta}\right)\right|_{\eta=\eta_{0}}$.

Proof. We write

$$
H_{\eta} u_{\eta}=v(\eta) u_{\eta}
$$

We have

$$
\left(H_{\eta}-v(\eta)\right) \partial_{\eta} u_{\eta}=\left(v^{\prime}(\eta)-\partial_{\eta} H_{\eta}\right) u_{\eta} .
$$

For $\eta=\eta_{0}$, this becomes

$$
\left.\left(H_{\eta_{0}}-v\left(\eta_{0}\right)\right)\left(\partial_{\eta} u_{\eta}\right)\right|_{\eta=\eta_{0}}=-\left.\left(\partial_{\eta} H_{\eta}\right)\right|_{\eta=\eta_{0}} u_{\eta_{0}} .
$$

The next lemma is sometimes called "effective mass theorem" (see [26]).

Lemma 1.5. We have

$$
\left(H_{\eta_{0}}-v\left(\eta_{0}\right)\right) w_{\eta_{0}}=\left(v^{\prime \prime}\left(\eta_{0}\right)-2\right) u_{\eta_{0}}-\left.2\left(\partial_{\eta} H_{\eta}\right)\right|_{\eta=\eta_{0}} v_{\eta_{0}},
$$

with $w_{\eta_{0}}=\left(\partial_{\eta}^{2} u_{\eta}\right)_{\eta=\eta_{0}}$. Moreover, we have

$$
\left\langle\left.\left(\partial_{\eta} H_{\eta}\right)\right|_{\eta=\eta_{0}} v_{\eta_{0}}, u_{\eta_{0}}\right\rangle=\frac{\nu^{\prime \prime}\left(\eta_{0}\right)-2}{2} .
$$

Proof. Taking the derivative of (1.5) with respect to $\eta$, we obtain

$$
\left(H_{\eta_{0}}-v\left(\eta_{0}\right)\right)\left(\partial_{\eta}^{2} u_{\eta}\right)_{\eta=\eta_{0}}=\left(v^{\prime \prime}\left(\eta_{0}\right)-2\right) u_{\eta_{0}}-\left.2\left(\partial_{\eta} H_{\eta}\right)\right|_{\eta=\eta_{0}} v_{\eta_{0}} .
$$

Then, we take the scalar product with $u_{\eta_{0}}$.

1.2. Local coordinates $(s, t)$. Before stating the main result of this paper, we shall introduce some notation related to the geometry of the zero locus of $\beta$. We use the local coordinates $(s, t)$, where $t(x)$ is the algebraic distance between $x$ and $\Gamma$ and $s(x)$ is the tangential coordinate of $x$. We choose a parametrization of $\Gamma$ :

$$
\gamma: \mathbb{R} /(|\partial \Omega| \mathbb{Z}) \longrightarrow \Gamma
$$


We choose the orientation of the parametrization $\gamma$ to be counter-clockwise, so that

$$
\operatorname{det}\left(\gamma^{\prime}(s), v(s)\right)=1 .
$$

The curvature $k(s)$ at the point $\gamma(s)$ is given in this parametrization by

$$
\gamma^{\prime \prime}(s)=k(s) v(s) .
$$

The map $\Phi$ defined by

$$
\begin{aligned}
\Phi: \mathbb{R} /(|\Gamma| \mathbb{Z}) \times\left(-t_{0}, t_{0}\right) & \longrightarrow \mathbb{R}^{2}, \\
(s, t) & \longmapsto \gamma(s)+t v(s),
\end{aligned}
$$

is clearly a diffeomorphism, when $t_{0}$ is sufficiently small, with image

$$
\Phi\left(\mathbb{R} /(|\Gamma| \mathbb{Z}) \times\left(-t_{0}, t_{0}\right)\right)=\left\{x \in \Omega \mid d(x, \Gamma)<t_{0}\right\}=\Omega_{t_{0}} .
$$

We let

$$
\begin{gathered}
\tilde{A}_{1}(s, t)=(1-t k(s)) \mathbf{A}(\Phi(s, t)) \cdot \gamma^{\prime}(s), \quad \tilde{A}_{2}(s, t)=\mathbf{A}(\Phi(s, t)) \cdot v(s), \\
\tilde{\beta}(s, t)=\beta(\Phi(s, t)),
\end{gathered}
$$

and we get

$$
\partial_{s} \tilde{A_{2}}-\partial_{t} \widetilde{A_{1}}=(1-t k(s)) \tilde{\beta}(s, t) .
$$

The quadratic form becomes

$$
\widetilde{Q}_{h, \mathbf{A}}(\psi)=\int(1-t k(s))\left|\left(-i h \partial_{t}+\widetilde{A}_{2}\right) \psi\right|^{2}+(1-t k(s))^{-1}\left|\left(-i h \partial_{s}+\widetilde{A}_{1}\right) \psi\right|^{2} d s d t .
$$

In a (simply connected) neighborhood of $(0,0)$, we can choose a gauge such that:

$$
\tilde{A}_{1}(s, t)=-\int_{0}^{t}\left(1-t^{\prime} k(s)\right) \tilde{\beta}\left(s, t^{\prime}\right) d t^{\prime}, \quad \tilde{A}_{2}=0 .
$$

1.3. Assumptions and main result. We consider the normal derivative of $\beta$ on $\Gamma$, i.e. the function $\delta: s \mapsto \partial_{t} \tilde{\beta}(s, 0)$. We will assume that:

$\delta$ admits a unique, non-degenerate and positive minimum at $x_{0}$.

We let $\delta_{0}=\delta(0)$ and assume without loss of generality that $x_{0}=(0,0)$. Let us state the main result of this paper.

Theorem 1.6. We assume (1.8). For all $n \geq 1$, there exist a sequence $\left(\theta_{j}^{n}\right)_{j \geq 0}$ and $h_{0}>0$ such that for $h \in\left(0, h_{0}\right)$, we have

$$
\lambda_{n}(h) \sim h^{4 / 3} \sum_{j \geq 0} \theta_{j}^{n} h^{j / 6}
$$


where

$$
\begin{aligned}
& \theta_{0}^{n}=\delta_{0}^{2 / 3} v_{1}\left(\eta_{0}\right) \\
& \theta_{1}^{n}=0 \\
& \theta_{2}^{n}=\delta_{0}^{2 / 3} C_{0}+\delta_{0}^{2 / 3}(2 n-1)\left(\frac{\alpha v\left(\eta_{0}\right) v^{\prime \prime}\left(\eta_{0}\right)}{3}\right)^{1 / 2}
\end{aligned}
$$

where we have let

$$
\alpha=\frac{1}{2} \delta_{0}^{-1} \delta^{\prime \prime}(0)>0
$$

and

$$
C_{0}=\left\langle L u_{\eta_{0}}, u_{\eta_{0}}\right\rangle_{\hat{\tau}}
$$

where

$$
L=2 \kappa(0) \delta_{0}^{-4 / 3}\left(\frac{\hat{\tau}^{2}}{2}-\eta_{0}\right) \hat{\tau}^{3}+2 \hat{\tau} \delta_{0}^{-1 / 3} k(0)\left(-\eta_{0}+\frac{\hat{\tau}^{2}}{2}\right)^{2}
$$

and

$$
\kappa(0)=\frac{1}{6} \partial_{t}^{2} \tilde{\beta}(0,0)-\frac{k(0)}{3} \delta_{0} .
$$

Let us make a few remarks concerning our main theorem and give perspectives.

Remark 1.7. This theorem is mainly motivated by the paper of B. Helffer and Y. Kordyukov [18] (see also [16], Section 5.2, where the result of this paper is presented as a conjecture and the paper [20] where the case of discrete wells is analyzed) where the authors prove a one term asymptotics for all the eigenvalues (see [18], Corollary 1.1). Moreover, they also prove an accurate upper bound in [18], Theorem 1.4, thanks to a Grushin type method (see [13]). In the present case (dimension 2 and the order of cancellation is $k=1$ ), our result is stronger in the sense that we get a complete asymptotics (in the same spirit as [11]).

Remark 1.8. As mentioned in the previous remark, in comparison with [18], we only deal with the case of dimension $2, k=1$ and when the metrics is flat. Nevertheless, the different generalizations are technical adaptations. Indeed, when $k=1$, in the case of higher dimension and with a Riemannian metrics, the only additional (and technical) point is the introduction of normal coordinates related to the Riemannian structure. After such a choice (using the exponential map), we are essentially reduced to the flat case (modulo error terms which are lower order) and our normal form technique can be implemented exactly in the same way. For the case $k \geq 1$, the difference is only the leading operator which is a higher order anharmonic oscillator (see [23]) and our method can again be used under the same kind of generic assumptions (see (1.8) where $\delta$ has to be replaced by $s \mapsto \partial_{t}^{k} \beta(s, 0)$ ). Let us finally mention that the case $k \geq 2$ and the cancellation along a hypersurface in dimension greater than 2 are maybe not the most generic situations. 
Remark 1.9. Theorem 1.6 can be seen as a semiclassical Birkhoff normal form for the magnetic Laplacian (see the references [39], [6], and [40] concerning the Birkhoff normal form).

In order to prove Theorem 1.6, it is enough to prove the two following theorems.

Theorem 1.10. We assume (1.8). For all $n \geq 1$, there exist a sequence $\left(\theta_{j}^{n}\right)_{j \geq 0}$ such that, for all $J \geq 0$, there exists $h_{0}>0$ such that for $h \in\left(0, h_{0}\right)$, we have

$$
d\left(h^{4 / 3} \sum_{j=0}^{J} \theta_{j}^{n} h^{j / 6}, \sigma\left(\mathscr{L}_{h, A}\right)\right) \leq C h^{4 / 3} h^{(J+1) / 6} .
$$

Moreover, we have

$$
\begin{aligned}
& \theta_{0}^{n}=\delta_{0}^{2 / 3} v_{1}\left(\eta_{0}\right), \\
& \theta_{1}^{n}=0, \\
& \theta_{2}^{n}=\delta_{0}^{2 / 3} C_{0}+\delta_{0}^{2 / 3}(2 n-1)\left(\frac{\alpha \nu\left(\eta_{0}\right) \nu^{\prime \prime}\left(\eta_{0}\right)}{3}\right)^{1 / 2} .
\end{aligned}
$$

The second theorem provides the spectral gap between the eigenvalues.

Theorem 1.11. We assume (1.8). For all $n \geq 1$, there exists $h_{0}>0$ such that for $h \in\left(0, h_{0}\right)$, we have

$$
\lambda_{n}(h) \geq h^{4 / 3}\left(\theta_{0}^{n}+h^{1 / 3} \theta_{2}^{n}\right)+o\left(h^{5 / 3}\right) .
$$

1.4. Organization of the paper. In Section 2, we prove Theorem 1.10. The main ingredients of the analysis are the Feynman-Hellmann theorem, the reduction of $\mathscr{L}_{h, \mathbf{A}}$ to a "normal form" and an expansion of the operator in power series which is an alternative to the so-called Grushin procedure (see [13], [16], and [11]). In Section 3, we prove localization and micro-localization estimates for the true eigenfunctions thanks to the Agmon estimates and a repeated use of the IMS formula. More precisely we will prove estimates of the eigenfunctions with respect to $s$ and $D_{s}$ in order to reduce the symbol of the operator when acting on the eigenfunctions. In Section 4, we use the localization results of Section 3 to estimate the Feshbach-Grushin projection and reduce the operator to a Schrödinger operator with electric potential which is in the Born-Oppenheimer form. Finally, we use the Born-Oppenheimer theory to estimate the spectral gap between the eigenvalues of Theorem 1.11.

Acknowledgments. N. Dombrowski acknowledges the partial support of "Nucleo Cientifico ICM P07-027-F: Mathematical Theory of Quantum and Classical Magnetic Systems" and also Georgi Raykov for his support. N. Raymond would like to thank F. Faure for giving the idea to write this paper and also B. Helffer for useful references. 


\section{Construction of quasimodes}

This section is devoted to the proof of Theorem 1.10.

2.1. Reduction to a normal form. Before starting the analysis, we shall use a few unitary transformations to normalize $\mathscr{L}_{h, \mathbf{A}}$. Let us notice that these transformations do not appear in [18] (or in the context of [11]) and permit to strongly simplify the analysis.

We can write the operator near the cancellation line in the coordinates $(s, t)$

$$
\tilde{\mathscr{L}}_{h, \mathbf{A}}=h^{2}(1-t k(s))^{-1} D_{t}(1-t k(s)) D_{t}+(1-t k(s))^{-1} \widetilde{P}(1-t k(s))^{-1} \widetilde{P},
$$

where

$$
\widetilde{P}=i h \partial_{s}+\widetilde{A}(s, t)
$$

with

$$
\widetilde{A}(s, t)=\int_{0}^{t}\left(1-k(s) t^{\prime}\right) \tilde{\beta}\left(s, t^{\prime}\right) d t^{\prime} .
$$

In terms of the quadratic form, we can write

$$
\tilde{Q}_{h, \mathbf{A}}(\psi)=\int\left(\left|h D_{t} \psi\right|^{2}+(1-t k(s))^{-2}|\tilde{P} \psi|^{2}\right) m(s, t) d s d t,
$$

with

$$
m(s, t)=(1-t k(s)) .
$$

We consider the following operator on $L^{2}(d s d t)$ which is unitarily equivalent to $\widetilde{\mathscr{L}}_{h, \mathrm{~A}}$ (see [27], Theorem 18.5.9 and below):

$$
\mathscr{L}_{h, \mathbf{A}}^{\text {new }}=m^{1 / 2} \tilde{\mathscr{L}}_{h, \mathbf{A}} m^{-1 / 2}=P_{1}^{2}+P_{2}^{2}-\frac{h^{2} k(s)^{2}}{4 m^{2}},
$$

with $P_{1}=m^{-1 / 2}\left(-h D_{s}+\widetilde{A}(s, t)\right) m^{-1 / 2}$ and $P_{2}=h D_{t}$.

We wish to use a system of coordinates more adapted to the magnetic situation. Let us perform a Taylor expansion near $t=0$. We have

$$
\tilde{\beta}(s, t)=\delta(s) t+\partial_{t}^{2} \tilde{\beta}(s, 0) \frac{t^{2}}{2}+O\left(t^{3}\right) .
$$

This provides

$$
\widetilde{A}(s, t)=\frac{\delta(s)}{2} t^{2}+\kappa(s) t^{3}+O\left(t^{4}\right),
$$

with

$$
\kappa(s)=\frac{1}{6} \partial_{t}^{2} \tilde{\beta}(s, 0)-\frac{k(s)}{3} \delta(s)
$$


This suggests, as for the model operator, to introduce the new magnetic coordinates in a fixed neighborhood of $(0,0)$,

$$
\tau=\delta(s)^{1 / 3} t, \quad \sigma=s .
$$

The change of coordinates for the derivatives is given by

$$
D_{t}=\delta(\sigma)^{1 / 3} D_{\tau}, \quad D_{s}=D_{\sigma}+\frac{1}{3} \delta^{\prime} \delta^{-1} \tau D_{\tau} .
$$

The space $L^{2}(d s d t)$ becomes $L^{2}\left(\delta(\sigma)^{-1 / 3} d \sigma d \tau\right)$. In the same way as previously, we shall conjugate $\mathscr{L}_{h, \mathbf{A}}^{\text {new }}$. We introduce the self-adjoint operator on $L^{2}(d \sigma d \tau)$

$$
\check{\mathscr{L}}_{h, \mathbf{A}}=\delta^{-1 / 6} \mathscr{L}_{h, \mathbf{A}}^{\text {new }} \delta^{1 / 6}
$$

We deduce

$$
\check{\mathscr{L}}_{h, \mathbf{A}}=h^{2} \delta(\sigma)^{2 / 3} D_{\tau}^{2}+\check{P}^{2},
$$

where

$$
\check{P}=\delta^{-1 / 6} \check{m}^{-1 / 2}\left(-h D_{\sigma}+\check{A}(\sigma, \tau)-h \frac{1}{3} \delta^{\prime} \delta^{-1} \tau D_{\tau}\right) \check{m}^{-1 / 2} \delta^{1 / 6},
$$

with

$$
\check{A}(\sigma, \tau)=\widetilde{A}\left(\sigma, \delta(\sigma)^{-1 / 3} \tau\right) .
$$

A straight forward computation provides

$$
\check{P}=\check{m}^{-1 / 2}\left(-h D_{\sigma}+\check{A}(\sigma, \tau)-h \frac{1}{6} \delta^{\prime} \delta^{-1}\left(\tau D_{\tau}+D_{\tau} \tau\right)\right) \check{m}^{-1 / 2},
$$

where we make the generator of dilations $\tau D_{\tau}+D_{\tau} \tau$ to appear (and which is related to the virial theorem). Up to a change of gauge, we can replace $\check{P}$ by

$$
\check{m}^{-1 / 2}\left(-h D_{\sigma}-\eta_{0}(\delta(\sigma))^{1 / 3} h^{2 / 3}+\check{A}(\sigma, \tau)-h \frac{1}{6} \delta^{\prime} \delta^{-1}\left(\tau D_{\tau}+D_{\tau} \tau\right)\right) \check{m}^{-1 / 2} .
$$

Normal form $\check{\mathscr{L}}(\boldsymbol{h})$. Therefore, the operator takes the form "à la Hörmander"

$$
\check{\check{L}}(h)=P_{1}(h)^{2}+P_{2}(h)^{2}-\frac{h^{2} k(\sigma)^{2}}{4 m\left(\sigma, \delta(\sigma)^{1 / 3} \tau\right)^{2}},
$$

where

$$
\begin{aligned}
& P_{1}(h)=\check{m}^{-1 / 2}(-h D_{\sigma}-\eta_{0}(\delta(\sigma))^{1 / 3} h^{2 / 3} \\
&\left.+\check{A}(\sigma, \tau)-h \frac{1}{6} \delta^{\prime} \delta^{-1}\left(\tau D_{\tau}+D_{\tau} \tau\right)\right) \check{m}^{-1 / 2}, \\
& P_{2}(h)=h \delta(\sigma)^{1 / 3} D_{\tau} .
\end{aligned}
$$


Computing a commutator, we can rewrite $P_{1}(h)$ as $P_{1}(h)=\check{m}^{-1}\left(-h D_{\sigma}-\eta_{0}(\delta(\sigma))^{1 / 3} h^{2 / 3}+\check{A}(\sigma, \tau)-h \frac{1}{6} \delta^{\prime} \delta^{-1}\left(\tau D_{\tau}+D_{\tau} \tau\right)\right)+C_{h}$,

where

$$
C_{h}=-h \check{m}^{-1 / 2}\left(D_{\sigma} \check{m}^{-1 / 2}\right)-\frac{h \delta^{\prime} \delta^{-1}}{3} \tau \check{m}^{-1 / 2}\left(D_{\tau} \check{m}^{-1 / 2}\right) .
$$

Notation 2.1. The quadratic form corresponding to $\check{\mathscr{L}}(h)$ will be denoted by $\check{Q}$.

Remark 2.2. The different transformations that we have used are allowed as soon as the functions of which acts $\mathscr{L}_{h, \mathbf{A}}$ are compactly supported near $\Gamma$. This will be the case for the quasimodes that we will use. Moreover in the localization analysis of the eigenfunctions, we will see that we will be able to truncate (with a rough support) the eigenfunctions by loosing a remainder of order $O\left(h^{\infty}\right)$.

Remark 2.3. As we will see in the analysis, the "normal form" given by (2.1) will spare us many technical considerations (see [11] and [18]) involved in the construction of quasimodes and also in the microlocal estimates.

2.2. Construction of quasimodes. We now enter in the proof of Theorem 1.10. The main ingredient for the proof is to homogenize the operator $\check{\mathscr{L}}$ and to use a formal power series expansion.

2.2.1. The homogenized operator $\hat{\mathscr{L}}$. We perform the scaling:

$$
\tau=h^{1 / 3} \hat{\tau}, \quad \sigma=h^{1 / 6} \hat{\sigma} .
$$

Notation 2.4. The operator $h^{-4 / 3} \check{\mathscr{L}}$ will be denoted by $\widehat{\mathscr{L}}$ in these new coordinates.

We expand the new operator in powers of $h^{1 / 6}$ in the sense of formal power series

$$
\delta_{0}^{-2 / 3} \hat{\mathscr{L}}(h) \sim \sum_{j \geq 0} \mathscr{L}_{j} h^{j / 6}
$$

with

$$
\begin{aligned}
& \mathscr{L}_{0}=D_{\hat{\tau}}^{2}+\left(-\eta_{0}+\frac{1}{2} \hat{\tau}^{2}\right)^{2}, \\
& \mathscr{L}_{1}=-2 D_{\hat{\sigma}}\left(-\eta_{0}+\frac{1}{2} \hat{\tau}^{2}\right), \\
& \mathscr{L}_{2}=D_{\hat{\sigma}}^{2}+\frac{2}{3} \alpha \hat{\sigma}^{2} \mathscr{L}_{0}+L,
\end{aligned}
$$


where $\alpha=\frac{1}{2} \delta_{0}^{-1} \delta^{\prime \prime}(0)>0$ and

$$
L=2 \kappa(0) \delta(0)^{-4 / 3}\left(\frac{\hat{\tau}^{2}}{2}-\eta_{0}\right) \hat{\tau}^{3}+2 \hat{\tau} \delta(0)^{-1 / 3} k(0)\left(-\eta_{0}+\frac{\hat{\tau}^{2}}{2}\right)^{2} .
$$

We look for quasi eigenpairs in the form

$$
\begin{array}{r}
\lambda \sim h^{4 / 3} \sum_{j \geq 0} \theta_{j} h^{j / 6}, \\
\psi \sim \sum_{j \geq 0} \psi_{j} h^{j / 6}
\end{array}
$$

so that, in the sense of formal power series

$$
\hat{\mathscr{L}}(h) \psi \sim \lambda \psi .
$$

2.2.2. Solving the formal system. Considering (2.4), we are led to solve an infinite formal system of PDE's which we will solve thanks a compatibility condition known as the Fredholm alternative.

Term in $\boldsymbol{h}^{\mathbf{0}}$. We solve the equation

$$
\mathscr{L}_{0} \psi_{0}=\theta_{0} \psi_{0} .
$$

This provides

$$
\theta_{0}=v_{1}\left(\eta_{0}\right)
$$

and

$$
\psi_{0}(\hat{\sigma}, \hat{\tau})=g_{0}(\hat{\sigma}) u_{\eta_{0}}(\hat{\tau})
$$

Term in $\boldsymbol{h}^{\mathbf{1 / 6}}$. We solve the equation

$$
\left(\mathscr{L}_{0}-\theta_{0}\right) \psi_{1}=\left(\theta_{1}-\mathscr{L}_{1}\right) \psi_{0} .
$$

Using Lemma 1.4, we have

$$
\left(\mathscr{L}_{0}-\theta_{0}\right)\left(\psi_{1}+D_{\hat{\sigma}} g_{0}(\hat{\sigma}) v_{\eta_{0}}(\hat{\tau})\right)=\theta_{1} \psi_{0} .
$$

The Fredholm alternative (the r. h. s. is orthogonal to $u_{\eta_{0}}$ for each $\hat{\sigma}$ ) implies

$$
\theta_{1}=0
$$

and

$$
\psi_{1}+D_{\hat{\sigma}} g_{0}(\hat{\sigma}) v_{\eta_{0}}(\hat{\tau})=g_{1}(\hat{\sigma}) u_{\eta_{0}}(\hat{\tau}),
$$

where $g_{1}$ shall be determined in a next step. 
Term in $\boldsymbol{h}^{2 / 6}$. We solve the equation

$$
\left(\mathscr{L}_{0}-\theta_{0}\right) \psi_{2}=\left(\theta_{2}-\mathscr{L}_{2}\right) \psi_{0}-\mathscr{L}_{1} \psi_{1}
$$

Using Lemmas 1.4 and 1.5, this equation rewrites

$$
\begin{aligned}
& \left(\mathscr{L}_{0}-\theta_{0}\right)\left(\psi_{2}+D_{\hat{\sigma}} g_{1} v_{\eta_{0}}-D_{\hat{\sigma}}^{2} g_{0} \frac{w_{\eta_{0}}}{2}\right) \\
& \quad=\left(\theta_{2} g_{0}-\frac{\nu^{\prime \prime}\left(\eta_{0}\right)}{2} D_{\hat{\sigma}}^{2} g_{0}-\frac{2}{3} \alpha \nu_{1}\left(\eta_{0}\right) \hat{\sigma}^{2} g_{0}-g_{0} L\left(\hat{\tau}, \partial_{\hat{\tau}}\right)\right) u_{\eta_{0}} .
\end{aligned}
$$

The Fredholm condition implies that, for all $\hat{\sigma}$,

$$
\left(\mathscr{H}+C_{0}\right) g_{0}=\theta_{2} g_{0},
$$

where $C_{0}$ is defined in (1.10) and where $\mathscr{H}$ denotes the effective harmonic oscillator (we recall (1.9) and that $\nu_{1}^{\prime \prime}\left(\eta_{0}\right)>0$ by (1.3)):

$$
\mathscr{H}=\frac{\nu^{\prime \prime}\left(\eta_{0}\right)}{2} D_{\hat{\sigma}}^{2}+\frac{2}{3} \alpha \hat{\sigma}^{2} .
$$

If we denote by $\left(\mu_{n}\right)_{n \geq 1}$ the increasing sequence of the eigenvalues of $\mathscr{H}$, we have by scaling

$$
\mu_{n}=(2 n-1)\left(\frac{\alpha v_{1}^{\prime \prime}\left(\eta_{0}\right)}{3}\right)^{1 / 2}
$$

Anyway we choose

$$
\theta_{2}=\mu_{n}+C_{0}
$$

and for $g_{0}$, we take $g_{(n)}$ a corresponding $L^{2}$-normalized eigenfunction. With theses choices, we determine a unique function $\psi_{2}^{\perp}$ which is solution of (2.5) and satisfying $\left\langle\psi_{2}^{\perp}, u_{\eta_{0}}\right\rangle_{\hat{\tau}}=0$ so that $\psi_{2}$ can be written as

$$
\psi_{2}=\psi_{2}^{\perp}-D_{\hat{\sigma}} g_{1} v_{\eta_{0}}+D_{\hat{\sigma}}^{2} g_{0} \frac{w_{\eta_{0}}}{2}+g_{2}(\hat{\sigma}) u_{\eta_{0}}(\hat{\tau}),
$$

where $g_{2}$ has to be determined in a next step.

Further terms ("Grushin procedure"). Let $J \geq 2$. Let us assume that $\psi_{0}, \ldots$, $\psi_{J-2}$ are determined as functions in the Schwartz class, that $\theta_{0}, \ldots, \theta_{J}$ are determined and that $\psi_{J-1}$ and $\psi_{J}$ are in the form

$$
\psi_{k}=\psi_{k}^{\perp}-D_{\hat{\sigma}} g_{k-1} v_{\eta_{0}}+g_{k} u_{\eta_{0}}, \quad k=J-1, J,
$$

where $\psi_{k}^{\perp}$ is a determined function in the Schwartz class which satisfies 


$$
\left\langle\psi_{k}^{\perp}, u_{\eta_{0}}\right\rangle_{\hat{\tau}}=0,
$$

for all $\hat{\sigma}$. Let us write the equation of order $J+1$ :

$$
\left(\mathscr{L}_{0}-\theta_{0}\right) \psi_{J+1}=\theta_{J+1} \psi_{0}+\sum_{j=2}^{J} \theta_{j} \psi_{J+1-j}-\sum_{j=1}^{J+1} \mathscr{L}_{j} \psi_{J+1-j}
$$

This equation can be put in the form

$$
\left(\mathscr{L}_{0}-\theta_{0}\right) \psi_{J+1}=\theta_{J+1} \psi_{0}+\theta_{2} \psi_{J-1}-\mathscr{L}_{1} \psi_{J}-\mathscr{L}_{2} \psi_{J-1}+F_{J},
$$

where $F_{J}$ is a determined function in the Schwartz class by recursion. We now use the explicit form of $\psi_{J-1}$ and $\psi_{J}$ and, using Lemmas 1.4 and 1.5, we deduce

$$
\begin{aligned}
& \left(\mathscr{L}_{0}-\theta_{0}\right)\left(\psi_{J+1}+D_{\hat{\sigma}} g_{J} v_{\eta_{0}}-D_{\hat{\sigma}}^{2} g_{J-1} \frac{w_{\eta_{0}}}{2}\right) \\
& \quad=\theta_{J+1} \psi_{0}+\left(\theta_{2} g_{J-1}-\frac{\nu^{\prime \prime}\left(\eta_{0}\right)}{2} D_{\hat{\sigma}}^{2} g_{J-1}-\frac{2}{3} \alpha \hat{\sigma}^{2} g_{J-1}-g_{J-1} L\right) u_{\eta_{0}}+\widetilde{F}_{J} .
\end{aligned}
$$

Taking the scalar product with $u_{\eta_{0}}$ with respect to the variable $\hat{\tau}$, we find the equation

$$
\left(\mathscr{H}+C_{0}\right) g_{J-1}-\theta_{2} g_{J-1}=\theta_{J+1} g_{(n)}+\left\langle\widetilde{F}_{J}, u_{\eta_{0}}\right\rangle_{\hat{\tau}},
$$

where $\mathscr{H}$ is given in (2.6). The Fredholm condition determines a unique pair $\left(\theta_{J+1}\right.$, $\left.g_{J-1}\right)$ with $g_{J-1}$ in the Schwartz class and such that $\left\langle g_{J-1}, g_{(n)}\right\rangle_{\hat{\sigma}}=0$.

Proof of Theorem 1.10. Let us introduce a smooth cutoff function $\chi_{0}$ supported in a fixed neighborhood of $x_{0}=(0,0)$. For $J \geq 0$ and $n \geq 1$, we let

$$
\psi_{h}^{[J, n]}=\chi_{0} \sum_{j=0}^{J} \psi_{j}\left(h^{-1 / 6} s, h^{-1 / 3} t\right) h^{j / 6},
$$

and

$$
\lambda_{h}^{[J, n]}=\delta_{0}^{2 / 3} h^{4 / 3} \sum_{j=0}^{J} \theta_{j} h^{j / 6}
$$

Using the fact the $\psi_{j}$ are in the Schwartz class, we deduce that

$$
\left\|\left(\check{\mathscr{L}}(h)-\lambda_{h}^{[J, n]}\right) \psi_{h}^{[J, n]}\right\| \leq C(J) h^{4 / 3} h^{(J+1) / 6}
$$

and the spectral theorem provides the conclusion. 


\section{Local and microlocal estimates}

This section deals with a priori estimates satisfied by the eigenfunctions of $\mathscr{L}_{h, \mathbf{A}}$.

3.1. A rough estimate for the eigenvalues. Let us first state an elementary lemma the proof of which can be found in [32], Theorem 5 .

Lemma 3.1. For all $\varphi \in \mathcal{C}_{0}^{\infty}\left(\mathbb{R}^{2}\right)$, we have

$$
Q_{h, \boldsymbol{A}}(\varphi) \geq\left.\left|\int_{\mathbb{R}^{2}} \beta(x)\right| \varphi\right|^{2} d x \mid .
$$

This lemma is interesting when the sign of $\beta$ does not change on the support of $\varphi$.

Proposition 3.2. For all $n \geq 1$, there exists $h_{0}>0$ such that, for $h \in\left(0, h_{0}\right)$ :

$$
\lambda_{n}(h) \geq \delta_{0}^{2 / 3} v_{1}\left(\eta_{0}\right) h^{4 / 3}-C h^{4 / 3+2 / 15} .
$$

Proof. We use a partition of unity with balls of size $h^{\rho}$

$$
\sum_{j} \chi_{j, h}^{2}=1
$$

and such that

$$
\sum\left|\nabla \chi_{j, h}\right|^{2} \leq C h^{-2 \rho}
$$

We will denote

$$
\mathscr{B}_{j, h}=\operatorname{supp} \chi_{j, h} .
$$

We have the IMS formula (cf. [8])

$$
\sum_{j} Q_{h, \mathbf{A}}\left(\chi_{j, h} \psi\right)-h^{2}\left\|\nabla \chi_{j, h} \psi\right\|^{2}=\lambda\left\|\chi_{j, h} \psi\right\|^{2} .
$$

We distinguish between the balls which intersect $t=0$ and the others so that we introduce

$$
J_{1}(h)=\left\{j: \mathscr{B}_{j, h} \cap \Gamma \neq \emptyset\right\} \quad \text { and } \quad J_{2}(h)=\left\{j: \mathscr{B}_{j, h} \cap \Gamma=\emptyset\right\} .
$$

If $j \in J_{2}(h)$, we use the inequality of Lemma 3.1,

$$
Q_{h, \mathbf{A}}\left(\chi_{j, h} \psi\right) \geq\left. h\left|\int \beta(x)\right| \chi_{j, h} \psi\right|^{2} d x \mid \geq c h^{1+\rho}\left\|\chi_{j, h} \psi\right\|^{2} .
$$

If $j \in J_{1}(h)$, we write

$$
Q_{h, \mathbf{A}}\left(\chi_{j, h} \psi\right) \geq\left(1-C h^{\rho}\right) \int\left|h \partial_{t}\left(\chi_{j, h} \psi\right)\right|^{2}+\left|\left(i h \partial_{s}+\tilde{A}\right)\left(\chi_{j, h} \psi\right)\right|^{2} d s d t,
$$


where we have

$$
\left|\widetilde{A}(s, t)-\frac{\delta\left(s_{j}\right) t^{2}}{2}\right| \leq C\left(t^{3}+\left|s-s_{j}\right| t^{2}\right) .
$$

We infer, for all $\varepsilon \in(0,1)$,

$$
\begin{aligned}
& Q_{h, \mathbf{A}}\left(\chi_{j, h} \psi\right) \\
& \geq\left(1-C h^{\rho}\right)\left((1-\varepsilon) \int\left|h \partial_{t}\left(\chi_{j, h} \psi\right)\right|^{2}+\mid\left(i h \partial_{s}\right.\right. \\
& \left.\left.\quad+\frac{\delta\left(s_{j}\right) t^{2}}{2}\right)\left.\left(\chi_{j, h} \psi\right)\right|^{2} d s d t-\frac{C h^{6 \rho}}{\varepsilon}\left\|\chi_{j, h} \psi\right\|^{2}\right),
\end{aligned}
$$

and we deduce, with (1.4),

$$
Q_{h, \mathbf{A}}\left(\chi_{j, h} \psi\right) \geq\left(1-C h^{\rho}\right)\left((1-\varepsilon) h^{4 / 3} v_{1}\left(\eta_{0}\right) \delta_{j}^{2 / 3}\left\|\chi_{j, h} \psi\right\|^{2}-\varepsilon^{-1} C h^{6 \rho}\left\|\chi_{j, h} \psi\right\|^{2}\right) .
$$

Optimizing with respect to $\varepsilon$, we choose

$$
\varepsilon=h^{3 \rho-\frac{2}{3}} .
$$

Then, we take $\rho$ such that

$$
2-2 \rho=3 \rho+\frac{2}{3}
$$

and we deduce

$$
\rho=\frac{4}{15}
$$

Jointly with Theorem 1.10 , we infer the following result.

Corollary 3.3. For all $n \geq 1$, we have

$$
\lambda_{n}(h)=\delta_{0}^{2 / 3} v_{1}\left(\eta_{0}\right) h^{4 / 3}+O\left(h^{4 / 3+2 / 15}\right) .
$$

3.2. Normal estimates of Agmon. In this subsection, we aim at proving localization estimates of Agmon type (cf. [1] and also [20], Section 5, and [22], Section 7, where the same ideas are used).

Proposition 3.4. Let $(\lambda, \psi)$ be an eigenpair of $\mathscr{L}_{h, A}$. There exist $h_{0}>0, C>0$ and $\varepsilon_{0}>0$ such that, for $h \in\left(0, h_{0}\right)$,

$$
\int e^{2 \varepsilon_{0}|t(x)| h^{-1 / 3}}|\psi|^{2} d x \leq C\|\psi\|^{2}
$$

and

$$
Q_{h, A}\left(e^{\varepsilon_{0}|t(x)| h^{-1 / 3}} \psi\right) \leq C h^{4 / 3}\|\psi\|^{2} .
$$


Proof. Let us consider an eigenpair $(\lambda, \psi)$ of $P_{h A}$. We begin to write the IMS formula:

$$
Q_{h, \mathbf{A}}\left(e^{\Phi} \psi\right)=\lambda\|\psi\|^{2}+h^{2}\left\|\nabla \Phi e^{\Phi} \psi\right\|^{2} .
$$

We use a partition of unity with balls of size $R h^{1 / 3}$

$$
\sum_{j} \chi_{j, h}^{2}=1
$$

and such that

$$
\sum\left|\nabla \chi_{j, h}\right|^{2} \leq C R^{-2} h^{-2 / 3}
$$

We may assume that the balls which intersect the line $t=0$ have their centers on it. Using again the IMS formula, we get the decomposition into local "energies"

$\sum_{j} Q_{h, \mathbf{A}}\left(\chi_{j, h} e^{\Phi} \psi\right)-\lambda\left\|\chi_{j, h} e^{\Phi} \psi\right\|^{2}-h^{2}\left\|\chi_{j, h} \nabla \Phi e^{\Phi} \psi\right\|^{2}-h^{2}\left\|\nabla \chi_{j, h} e^{\Phi} \psi\right\|^{2}=0$.

We distinguish between the balls which intersect $t=0$ and the others:

$$
J_{1}(h)=\left\{j: \mathscr{B}_{j, h} \cap \Gamma \neq \varnothing\right\}, \quad J_{2}(h)=\left\{j: \mathscr{B}_{j, h} \cap \Gamma=\emptyset\right\} .
$$

If $j \in J_{2}(h)$, we use Lemma 3.1 combined with the non-degeneracy of the cancellation of $\beta$ (see (1.8) in which we just use the positivity of the minimum) and 1.1. We get the existence of $c>0$ and $h_{0}>0$ such that, for $h \in\left(0, h_{0}\right)$,

$$
Q_{h, \mathbf{A}}\left(\chi_{j, h} e^{\Phi} \psi\right) \geq\left. h\left|\int \beta(x)\right| \chi_{j, h} e^{\Phi} \psi\right|^{2} d x \mid \geq c R h^{4 / 3}\left\|\chi_{j, h} e^{\Phi} \psi\right\|^{2} .
$$

If $j \in J_{1}(h)$, we write, in the same way as in the proof of Proposition 3.2,

$$
Q_{h, \mathbf{A}}\left(\chi_{j, h} e^{\Phi} \psi\right) \geq\left(1-C R h^{1 / 3}\right)\left((1-\varepsilon) h^{4 / 3} v_{1}\left(\eta_{0}\right) \delta_{j}^{2 / 3}-\varepsilon^{-1} C h^{2}\left\|\mid \chi_{j, h} e^{\Phi} \psi\right\|^{2}\right) .
$$

We take $\varepsilon=h^{1 / 3}$. We use Corollary 3.3 to get an upper bound on $\lambda$. We are led to choose $\Phi(x)=\varepsilon_{0}|t(x)| h^{-1 / 3}$ so that

$$
h^{2}|\nabla \Phi|^{2} \leq h^{4 / 3} \varepsilon_{0}^{2} .
$$

Taking $\varepsilon_{0}$ small enough and $R$ large enough, we infer the existence of $\tilde{c}>0, C>0$ and $h_{0}>0$ such that, for $h \in\left(0, h_{0}\right)$,

$$
\tilde{c} h^{4 / 3} \sum_{j \in J_{1}(h)} \int e^{2 \Phi}\left|\chi_{j, h} \psi\right|^{2} d x \leq C h^{4 / 3} \sum_{j \in J_{2}(h)} \int e^{2 \Phi}\left|\chi_{j, h} \psi\right|^{2} d x .
$$

Then, due to the support of $\chi_{j, h}$ when $j \in J_{2}(h)$,we infer

$$
\sum_{j \in J_{2}(h)} \int e^{2 \Phi}\left|\chi_{j, h} \psi\right|^{2} d x \leq \widetilde{C} \sum_{j \in J_{2}(h)} \int\left|\chi_{j, h} \psi\right|^{2} d x .
$$

We deduce (3.1). Finally, (3.2) follows from (3.1) and (3.3). 
3.3. Rough localization. This subsection is devoted to the proof of localization estimates near and on the cancellation line of the magnetic field.

Proposition 3.5. Let $(\lambda, \psi)$ be an eigenpair of $\mathscr{L}_{h, A}$. There exist $h_{0}>0, C>0$ and $\varepsilon_{0}>0$ such that, for $h \in\left(0, h_{0}\right)$,

$$
\int e^{2 \chi(t(x))|s(x)| h^{-1 / 15}}|\psi|^{2} d x \leq C\|\psi\|^{2}
$$

and

$$
Q_{h, A}\left(e^{\chi(t(x))|s(x)| h^{-1 / 15}} \psi\right) \leq C h^{4 / 3}\|\psi\|^{2},
$$

where $\chi$ is a fixed smooth cutoff function being 1 near 0 .

Proof. Let us consider an eigenpair $(\lambda, \psi)$ of $P_{h, \mathbf{A}}$. We begin to write the IMS formula

$$
Q_{h, \mathbf{A}}\left(e^{\Phi} \psi\right)=\lambda\|\psi\|^{2}+h^{2}\left\|\nabla \Phi e^{\Phi} \psi\right\|^{2} .
$$

We use a partition of unity with balls of size $h^{4 / 15}$

$$
\sum_{j} \chi_{j, h}^{2}=1
$$

and such that

$$
\sum\left|\nabla \chi_{j, h}\right|^{2} \leq C h^{-8 / 15}
$$

We take

$$
\Phi=\chi(t(x))|s(x)| h^{-1 / 15}
$$

In particular, we have

$$
|\nabla \Phi| \leq C h^{-1 / 15}
$$

We write

$$
\sum_{j} Q_{h, \mathbf{A}}\left(\chi_{j, h} e^{\Phi} \psi\right)-\lambda\left\|\chi_{j, h} e^{\Phi} \psi\right\|^{2}-h^{2}\left\|\chi_{j, h} \nabla \Phi e^{\Phi} \psi\right\|^{2}-h^{2}\left\|\nabla \chi_{j, h} e^{\Phi} \psi\right\|^{2}=0 .
$$

Let us defined the two subsets of index

$$
J_{1}(h)=\left\{j: \mathscr{B}_{j, h} \cap \Gamma \neq \emptyset\right\} \quad \text { and } \quad J_{2}(h)=\left\{j: \mathscr{B}_{j, h} \cap \Gamma=\emptyset\right\} .
$$

As previously, we can write, for the balls with index $j \in J_{2}(h)$,

$$
Q_{h, \mathbf{A}}\left(\chi_{j, h} e^{\Phi} \psi\right) \geq\left. h\left|\int \beta(x)\right| \chi_{j, h} e^{\Phi} \psi\right|^{2} d x \mid \geq c h^{1+4 / 15}\left\|\chi_{j, h} e^{\Phi} \psi\right\|^{2} .
$$

For the balls of index $j \in J_{1}(h)$, we have

$$
Q_{h, \mathbf{A}}\left(\chi_{j, h} e^{\Phi} \psi\right) \geq\left(h^{4 / 3} v_{1}\left(\eta_{0}\right) \delta_{j}^{2 / 3}-C h^{4 / 3+2 / 15}\right)\left\|\chi_{j, h} e^{\Phi} \psi\right\|^{2},
$$


where $\delta_{j}=\delta\left(s_{j}\right)\left(\left(s_{j}, 0\right)\right.$ is the center of the ball). Gathering the estimates, we deduce

$$
\begin{aligned}
& \left(c h^{1+4 / 15}-h^{4 / 3} v_{1}\left(\eta_{0}\right) \delta_{0}^{2 / 3}\right) \sum_{j \in J_{2}(h)}\left\|\chi_{j, h} e^{\Phi} \psi\right\|^{2} \\
& \quad+\sum_{j \in J_{1}(h)}\left(h^{4 / 3} v_{1}\left(\eta_{0}\right)\left(\delta_{j}^{2 / 3}-\delta_{0}^{2 / 3}\right)-C h^{4 / 3+2 / 15}\right)\left\|\chi_{j, h} e^{\Phi} \psi\right\|^{2} \\
& \quad \leq 0 .
\end{aligned}
$$

Then, we fix $\varepsilon_{0}>0$ and $D>0$ and we write

$$
J_{1}(h)=J_{1,1}(h) \cup J_{1,2}(h) \cup J_{1,3}(h),
$$

where

$$
\begin{aligned}
& J_{1,1}(h)=\left\{j \in J_{1}(h):\left|s_{j}\right| \leq D h^{1 / 15}\right\}, \\
& J_{1,2}(h)=\left\{j \in J_{1}(h): D h^{1 / 15}<\left|s_{j}\right| \leq \varepsilon_{0}\right\}, \\
& J_{1,3}(h)=\left\{j \in J_{1}(h):\left|s_{j}\right| \geq \varepsilon_{0}\right\} .
\end{aligned}
$$

For $j \in J_{1,3}(h)$, there exist $c\left(\varepsilon_{0}\right)>0$ and $h_{0}>0$ such that, for $h \in\left(0, h_{0}\right)$,

$$
h^{4 / 3} v_{1}\left(\eta_{0}\right)\left(\delta_{j}^{2 / 3}-\delta_{0}^{2 / 3}\right)-C h^{4 / 3+2 / 15} \geq c\left(\varepsilon_{0}\right) h^{4 / 3} .
$$

For $j \in J_{1,2}(h)$, from the assumption 1.8 , there exists $\tilde{c}\left(\varepsilon_{0}\right)>0$ such that

$$
h^{4 / 3} v_{1}\left(\eta_{0}\right)\left(\delta_{j}^{2 / 3}-\delta_{0}^{2 / 3}\right)-C h^{4 / 3+2 / 15} \geq h^{4 / 3} \tilde{c}\left(\varepsilon_{0}\right) s_{j}^{2}-C h^{4 / 3+2 / 15} .
$$

We notice that

$$
h^{4 / 3} \tilde{c}\left(\varepsilon_{0}\right) s_{j}^{2}-C h^{4 / 3+2 / 15} \geq h^{4 / 3}\left(\tilde{c}\left(\varepsilon_{0}\right) D^{2}-C\right) h^{2 / 15} .
$$

We choose $D$ so that

$$
\tilde{c}\left(\varepsilon_{0}\right) D^{2}-C>0 .
$$

We notice that, for $j \in J_{1,1}(h)$,

$$
\left\|\chi_{j, h} e^{\Phi} \psi\right\| \leq C\|\psi\|
$$

We now deduce from (3.7)

$$
\sum_{j \in J_{1,2}(h)}\left\|\chi_{j, h} e^{\Phi} \psi\right\|^{2} \leq C\|\psi\|^{2}
$$

and then

$$
\sum_{j \in J_{1,3}(h)}\left\|\chi_{j, h} e^{\Phi} \psi\right\|^{2} \leq C\|\psi\|^{2}, \quad \sum_{j \in J_{2}(h)}\left\|\chi_{j, h} e^{\Phi} \psi\right\|^{2} \leq C\|\psi\|^{2} .
$$

This provides (3.4) and the identity (3.6) implies (3.5). 
Introduction of cutoff functions. From Propositions 3.4 and 3.5, we are led to introduce a cutoff function living near $x_{0}$. We take $\gamma>0$ and we let

$$
\chi_{h, \gamma}(x)=\chi\left(h^{-1 / 3+\gamma} t(x)\right) \chi\left(h^{-1 / 15+\gamma} s(x)\right) .
$$

where $\chi$ is a fixed smooth cutoff function supported near 0 . Moreover, we will denote by $\check{\psi}$ the function $\chi_{h, \gamma}(x) \psi(x)$ in the coordinates $(\sigma, \tau)$. In particular, we have

$$
\|\check{\psi}\|=\left(1+O\left(h^{\infty}\right)\right)\|\psi\| .
$$

As a consequence of Proposition 3.4 we have the following corollary.

Corollary 3.6. Let $(\lambda, \psi)$ be an eigenpair of $\mathscr{L}_{h, A}$. For all $n \in \mathbb{N}$, there exist $h_{0}>0$, $C>0$ and $\varepsilon_{0}>0$ such that, for $h \in\left(0, h_{0}\right)$,

$$
\int \tau^{n}|\check{\psi}|^{2} d \sigma d \tau \leq C h^{2 n / 3}\|\check{\psi}\|^{2}
$$

and

$$
\int \tau^{n}\left(\left|h D_{\tau} \check{\psi}\right|^{2}+\left|h D_{\sigma} \check{\psi}\right|^{2}\right) d \sigma d \tau \leq C h^{2 n / 3} h^{4 / 3}\|\check{\psi}\|^{2} .
$$

3.4. Order of the second term. From the normal estimates of Agmon, we deduce the following proposition.

Proposition 3.7. For all $n \geq 1$, there exist $h_{0}>0$ and $C>0$ such that, for $h \in\left(0, h_{0}\right)$,

$$
\lambda_{n}(h) \geq \delta_{0}^{2 / 3} v_{1}\left(\eta_{0}\right) h^{4 / 3}-C h^{5 / 3} .
$$

Proof. We consider an eigenpair $\left(\lambda_{n}(h), \psi_{n, h}\right)$ and we use the IMS formula

$$
\check{Q}\left(\check{\psi}_{n, h}\right)=\lambda_{n}(h)\left\|\check{\psi}_{n, h}\right\|^{2}+O\left(h^{\infty}\right)\left\|\check{\psi}_{n, h}\right\|^{2} .
$$

We have (cf. (2.1)),

$$
\begin{aligned}
& \check{Q}\left(\check{\psi}_{n, h}\right) \\
& \geq \int \check{m}^{-2} \|\left(-h D_{\sigma}-\eta_{0} \delta^{1 / 3} h^{2 / 3}\right. \\
& \left.+\check{A}-\frac{h}{6} \delta^{\prime} \delta^{-1}\left(\tau D_{\tau}+D_{\tau} \tau\right)+C_{h}\right)\left.\check{\psi}_{n, h}\right|^{2} d \sigma d \tau \\
& +h^{2} \delta^{2 / 3}\left\|D_{\tau} \check{\psi}_{n, h}\right\|^{2}-C h^{2}\left\|\check{\psi}_{n, h}\right\|^{2} .
\end{aligned}
$$

Let us deal with the terms involving $C_{h}$ in the double product produced by the expansion of the square. We have to estimate

$$
h\left|\operatorname{Re}\left\langle\delta^{\prime} \delta^{-1}\left(\tau D_{\tau}+D_{\tau} \tau\right) \check{\psi}_{n, h}, C_{h} \check{\psi}_{n, h}\right\rangle\right| .
$$


We have

$$
\left\|C_{h} \breve{\psi}_{n, h}\right\|=o(h)\left\|\check{\psi}_{n, h}\right\|
$$

and, with the estimates of Agmon (and the fact that 0 is a critical point of $\delta$ ),

$$
\left\|\delta^{\prime} \delta^{-1}\left(\tau D_{\tau}+D_{\tau} \tau\right) \check{\psi}_{n, h}\right\|=o(1)\left\|\check{\psi}_{n, h}\right\| .
$$

Moreover, we have in the same way

$$
h\left|\operatorname{Re}\left\langle\check{A}_{\bar{\psi}}, h, C_{h} \check{\psi}_{n, h}\right\rangle\right|=o\left(h^{5 / 3}\right)\left\|\check{\psi}_{n, h}\right\|^{2} .
$$

Then, we have the control

$$
h\left|\operatorname{Re}\left\langle\check{h}_{\sigma} \check{\psi}_{n, h}, C_{h} \check{\psi}_{n, h}\right\rangle\right|=o\left(h^{5 / 3}\right)\left\|\check{\psi}_{n, h}\right\|^{2},
$$

where we have used the rough estimate

$$
\left\|h D_{\sigma} \check{\psi}_{n, h}\right\| \leq C h^{2 / 3}\left\|\check{\psi}_{n, h}\right\| .
$$

We have

$$
\begin{aligned}
& \check{Q}\left(\check{\psi}_{n, h}\right) \\
& \geq \int \check{m}^{-2}\left|\left(-h D_{\sigma}-\eta_{0} \delta^{1 / 3} h^{2 / 3}+\check{A}-\frac{h}{6} \delta^{\prime} \delta^{-1}\left(\tau D_{\tau}+D_{\tau} \tau\right)\right) \check{\psi}_{n, h}\right|^{2} d \sigma d \tau \\
& \quad+h^{2} \delta_{0}^{2 / 3}\left\|D_{\tau} \check{\psi}_{n, h}\right\|^{2}+o\left(h^{5 / 3}\right)\left\|\check{\psi}_{n, h}\right\|^{2} .
\end{aligned}
$$

We now deal with the term involving $\tau D_{\tau}+D_{\tau} \tau$. With the estimates of Agmon, we have

$h\left|\operatorname{Re}\left\langle\delta^{\prime} \delta^{-1}\left(\tau D_{\tau}+D_{\tau} \tau\right) \check{\psi}_{n, h},\left(-h D_{\sigma}-\eta_{0} \delta^{1 / 3} h^{2 / 3}+\check{A}\right) \check{\psi}_{n, h}\right\rangle\right|=o\left(h^{5 / 3}\right)\left\|\breve{\psi}_{n, h}\right\|^{2}$.

This implies

$$
\begin{aligned}
& \check{Q}\left(\check{\psi}_{n, h}\right) \\
& \geq \delta_{0}^{2 / 3} h^{2}\left\|D_{\tau} \check{\psi}_{n, h}\right\|^{2}+\int \check{m}^{-2} \mid\left(-h D_{\sigma}-\eta_{0} \delta^{1 / 3} h^{2 / 3}+\left.\check{A}^{2} \check{\psi}_{n, h}\right|^{2} d \sigma d \tau\right. \\
& \quad+o\left(h^{5 / 3}\right)\left\|\check{\psi}_{n, h}\right\|^{2} .
\end{aligned}
$$

With the same arguments, it follows

$$
\begin{aligned}
& \check{Q}\left(\check{\psi}_{n, h}\right) \\
& \geq h^{2} \delta_{0}^{2 / 3}\left\|D_{\tau} \check{\psi}_{n, h}\right\|^{2} \\
& \quad+\int \check{m}^{-2}\left|\left(-h D_{\sigma}-\eta_{0} \delta^{1 / 3} h^{2 / 3}+\delta^{1 / 3} \frac{\tau^{2}}{2}\right) \check{\psi}_{n, h}\right|^{2} d \sigma d \tau \\
& \quad+O\left(h^{5 / 3}\right)\left\|\check{\psi}_{n, h}\right\|^{2}
\end{aligned}
$$


and

$$
\begin{aligned}
& \check{Q}\left(\check{\psi}_{n, h}\right) \\
& \geq h^{2} \delta_{0}^{2 / 3}\left\|D_{\tau} \check{\psi}_{n, h}\right\|^{2}+\int\left|\left(-h D_{\sigma}-\eta_{0} \delta^{1 / 3} h^{2 / 3}+\delta^{1 / 3} \frac{\tau^{2}}{2}\right) \check{\psi}_{n, h}\right|^{2} d \sigma d \tau \\
& \quad+O\left(h^{5 / 3}\right)\left\|\check{\psi}_{n, h}\right\|^{2} .
\end{aligned}
$$

We get

$$
\begin{aligned}
& \check{Q}\left(\check{\psi}_{n, h}\right) \\
& \geq h^{2} \delta_{0}^{2 / 3}\left\|D_{\tau} \check{\psi}_{n, h}\right\|^{2}+\int \delta_{0}^{2 / 3}\left|\left(-h \delta^{-1 / 3} D_{\sigma}-\eta_{0} h^{2 / 3}+\frac{\tau^{2}}{2}\right) \check{\psi}_{n, h}\right|^{2} d \sigma d \tau \\
& \quad+O\left(h^{5 / 3}\right)\left\|\check{\psi}_{n, h}\right\|^{2} .
\end{aligned}
$$

Then, we write

$$
\delta^{-1 / 3} D_{\sigma}=\delta^{-1 / 6} D_{\sigma} \delta^{-1 / 6}+i\left(\delta^{-1 / 6}\right)^{\prime}
$$

and deduce

$$
\begin{aligned}
\check{Q}\left(\check{\psi}_{n, h}\right) \\
\geq h^{2} \delta_{0}^{2 / 3}\left\|D_{\tau} \check{\psi}_{n, h}\right\|^{2} \\
\quad+\int \delta_{0}^{2 / 3}\left|\left(-h \delta^{-1 / 6} D_{\sigma} \delta^{-1 / 6}-\eta_{0} h^{2 / 3}+\frac{\tau^{2}}{2}\right) \check{\psi}_{n, h}\right|^{2} d \sigma d \tau \\
\quad+O\left(h^{5 / 3}\right)\left\|\check{\psi}_{n, h}\right\|^{2} .
\end{aligned}
$$

We can apply the functional calculus to the self-adjoint operator $\delta^{-1 / 6} D_{\sigma} \delta^{-1 / 6}$ and the following lower bound follows:

$$
\check{Q}\left(\check{\psi}_{n, h}\right) \geq h^{4 / 3} \delta_{0}^{2 / 3} v_{1}\left(\eta_{0}\right)+O\left(h^{5 / 3}\right)\left\|\check{\psi}_{n, h}\right\|^{2} .
$$

From this analysis, we infer that, for all $n \geq 1$, there exist $h_{0}>0$ and $C>0$ such that, for all $h \in\left(0, h_{0}\right)$ :

$$
\left|\lambda_{n}(h)-\delta_{0}^{2 / 3} v_{1}\left(\eta_{0}\right) h^{2 / 3}\right| \leq C h^{5 / 3} .
$$

Introduction of the space generated by the truncated eigenfunctions. For all $N \geq 1$, let us consider $L^{2}$-normalized eigenpairs $\left(\lambda_{n}(h), \psi_{n, h}\right)_{1 \leq n \leq N}$ such that $\left\langle\psi_{n, h}, \psi_{m, h}\right\rangle=0$ if $n \neq m$. We consider the $N$ dimensional space defined by:

$$
\mathfrak{E}_{N}(h)=\operatorname{span}_{1 \leq n \leq N} \check{\psi}_{n, h} .
$$

Remark 3.8. The estimates of Agmon of Corollary 3.6 are satisfied by all the elements of $\mathfrak{E}_{N}(h)$. 
3.5. Localization with respect to $\sigma$ and $\boldsymbol{D}_{\boldsymbol{\sigma}}$. This subsection is devoted the analysis of the behavior of the eigenfunctions with respect to $\sigma$ and $D_{\sigma}$. In particular the crucial propositions that we prove are Propositions 3.9 and 3.12. We will see that these local and microlocal controls will be enough to estimate the spectral gap between the eigenvalues (we do not need higher order controls, i.e. estimates of $\sigma^{m}$ and $D_{\sigma}^{m}$, even if they could be proved).

3.5.1. Localization with respect to $\sigma$. This subsection deals with the proof of the following proposition.

Proposition 3.9. There exist $h_{0}>0, C>0$ such that, for $h \in\left(0, h_{0}\right)$ and for all $\check{\psi} \in \mathfrak{E}_{N}(h)$,

$$
\|\sigma \check{\psi}\| \leq C h^{1 / 6}\|\check{\psi}\| .
$$

Proof. We only have to prove the wished inequality for $\breve{\psi}^{\prime}=\check{\psi}_{n, h}$, the extension to $\check{\psi} \in \mathfrak{E}_{N}(h)$ being an easy consequence. We consider $(\lambda, \psi)$ an eigenpair of $\mathscr{L}_{h, \mathbf{A}}$. We can write

$$
\check{Q}(\check{\psi})=\lambda\|\check{\psi}\|^{2}+O\left(h^{\infty}\right)\|\check{\psi}\|^{2} .
$$

We have

$$
\check{Q}(\check{\psi})=\left\|P_{1}(h) \check{\psi}\right\|^{2}+\left\|P_{2}(h) \check{\psi}\right\|^{2}+O\left(h^{2}\right)\|\check{\psi}\|^{2} .
$$

We can write

$$
\begin{aligned}
& \left\|P_{1}(h) \check{\psi}\right\|^{2} \\
& =\| \check{m}^{-1}\left(-h D_{\sigma}-\eta_{0}(\delta(\sigma))^{1 / 3} h^{2 / 3}\right. \\
& \quad+\check{A}(\sigma, \tau)-h \frac{1}{6} \delta^{\prime} \delta^{-1}\left(\tau D_{\tau}+D_{\tau} \tau\right) \check{\psi}+C_{h} \check{\psi} \|^{2},
\end{aligned}
$$

with

$$
C_{h}=i h \check{m}^{-1 / 2} \partial_{\sigma} \check{m}^{-1 / 2}-\frac{h \delta^{\prime}}{6 \delta}\left[\tau D_{\tau}+D_{\tau} \tau, \check{m}^{-1 / 2}\right] .
$$

Let us first erase the term involving $C(h)$. From the estimates of Agmon, it follows

$$
\begin{aligned}
& \left\|P_{1}(h) \check{\psi}\right\|^{2} \\
& \quad \geq \| \check{m}-1\left(-h D_{\sigma}-\eta_{0}(\delta(\sigma))^{1 / 3} h^{2 / 3}+\check{A}(\sigma, \tau)-h \frac{1}{6} \delta^{\prime} \delta^{-1}\left(\tau D_{\tau}+D_{\tau} \tau\right) \check{\psi} \|^{2}\right. \\
& \quad-C h^{2}\|\check{\psi}\|^{2} .
\end{aligned}
$$

Then, we use again the normal Agmon estimates to replace $\check{m}$ by 1 :

$$
\begin{aligned}
\left\|P_{1}(h) \check{\psi}\right\|^{2} \\
\geq \\
\qquad\left(-h D_{\sigma}-\eta_{0}(\delta(\sigma))^{1 / 3} h^{2 / 3}+\check{A}(\sigma, \tau)-h \frac{1}{6} \delta^{\prime} \delta^{-1}\left(\tau D_{\tau}+D_{\tau} \tau\right) \check{\psi} \|^{2}\right. \\
\quad-C h^{5 / 3}\|\check{\psi}\|^{2} .
\end{aligned}
$$


From the non-degeneracy assumption on $\delta$, we infer that there exist $c>0$ and $h_{0}>0$ such that, for $h \in\left(0, h_{0}\right)$,

$\check{Q}(\check{\psi})$

$$
\begin{aligned}
& \geq \delta_{0}^{2 / 3} v_{1}\left(\eta_{0}\right) h^{4 / 3}\|\check{\psi}\|^{2}+c\left\|h D_{\tau} \sigma \check{\psi}\right\|^{2} \\
& \quad+c \int \sigma^{2} \mid\left(\left(i h \delta^{-1 / 3} \partial_{\sigma}+\eta_{0} h^{2 / 3}\right.\right. \\
& \left.\quad+\frac{\tau^{2}}{2}+R_{h}-\frac{h \delta^{\prime}}{6 \delta^{4 / 3}}\left(\tau D_{\tau}+D_{\tau} \tau\right)\right)\left.\check{\psi}\right|^{2} d \sigma d \tau \\
& \quad-C h^{2}\|\check{\psi}\|^{2},
\end{aligned}
$$

with

$$
R_{h}=\kappa(\sigma) \delta^{-4 / 3} \tau^{3}+O\left(\tau^{4}\right)
$$

We must analyze

$$
\begin{gathered}
\int \sigma^{2} \mid\left(\left.\left(i h \delta^{-1 / 3} \partial_{\sigma}+\eta_{0} h^{2 / 3}+\frac{\tau^{2}}{2}+R_{h}-\frac{h \delta^{\prime}}{6 \delta^{4 / 3}}\left(\tau D_{\tau}+D_{\tau} \tau\right)\right) \check{\psi}\right|^{2} d \sigma d \tau\right. \\
=\int \mid\left(\left(i h \delta^{-1 / 3} \partial_{\sigma}+\eta_{0} h^{2 / 3}\right.\right. \\
\left.\quad+\frac{\tau^{2}}{2}+R_{h}-\frac{h \delta^{\prime}}{6 \delta^{4 / 3}}\left(\tau D_{\tau}+D_{\tau} \tau\right)\right) \sigma \check{\psi}-\left.i h \delta^{-1 / 3} \check{\psi}\right|^{2} d \sigma d \tau .
\end{gathered}
$$

We shall estimate the double product

$$
\begin{array}{r}
2 \int \operatorname{Re}\left\{i \left(\left(i h \delta^{-1 / 3} \partial_{\sigma}+\eta_{0} h^{2 / 3}\right.\right.\right. \\
\left.\left.+\frac{\tau^{2}}{2}+R_{h}-\frac{h \delta^{\prime}}{6 \delta^{4 / 3}}\left(\tau D_{\tau}+D_{\tau} \tau\right)\right) \sigma \check{\psi} \overline{h \delta^{-1 / 3} \check{\psi}}\right\} d \sigma d \tau \\
=2 h \int \operatorname{Re}\left\{i\left(i h \delta^{-1 / 3} \partial_{\sigma}-\frac{h \delta^{\prime}}{6 \delta^{4 / 3}}\left(\tau D_{\tau}+D_{\tau} \tau\right)\right) \sigma \check{\psi} \overline{\delta^{-1 / 3} \check{\psi}}\right\} d \sigma d \tau .
\end{array}
$$

We have

$$
\begin{aligned}
& -2 h^{2} \int \operatorname{Re}\left\{\delta^{-1 / 3} \partial_{\sigma}(\sigma \check{\psi}) \bar{\psi}\right\} d \sigma d \tau \\
& =O\left(h^{2}\right)\|\check{\psi}\|^{2}-h^{2} \int \delta^{-1 / 3} \partial_{\sigma}|\check{\psi}|^{2} d \sigma d \tau \\
& =O\left(h^{2}\right)\|\check{\psi}\|^{2} .
\end{aligned}
$$

Then, we write, thanks to the estimates of Agmon,

$$
-2 h^{2} \int \frac{\sigma \delta^{-1 / 3} \delta^{\prime}}{6 \delta^{4 / 3}} \operatorname{Re}\left\{\left(\left(\tau \partial_{\tau}+\partial_{\tau} \tau\right)\right) \check{\psi} \bar{\psi}\right\} d \sigma d \tau=O\left(h^{2}\right)\|\check{\psi}\|^{2} .
$$


We infer that

$$
\begin{aligned}
& \int \sigma^{2} \mid\left(\left.\left(i h \delta^{-1 / 3} \partial_{\sigma}+\eta_{0} h^{2 / 3}+\frac{\tau^{2}}{2}+R_{h}-\frac{h \delta^{\prime}}{6 \delta^{4 / 3}}\left(\tau D_{\tau}+D_{\tau} \tau\right)\right) \check{\psi}\right|^{2} d \sigma d \tau\right. \\
& =\int \mid\left(\left.\left(i h \delta^{-1 / 3} \partial_{\sigma}+\eta_{0} h^{2 / 3}+\frac{\tau^{2}}{2}+R_{h}-\frac{h \delta^{\prime}}{6 \delta^{4 / 3}}\left(\tau D_{\tau}+D_{\tau} \tau\right)\right) \sigma \check{\psi}\right|^{2} d \sigma d \tau\right. \\
& \quad+O\left(h^{2}\right)\|\check{\psi}\|^{2} .
\end{aligned}
$$

We have to control the following double product:

$$
\frac{2}{6} h^{2} \operatorname{Re}\left\{\int i \delta^{-1 / 3} \partial_{\sigma}(\sigma \check{\psi}) \overline{\delta^{-4 / 3} \delta^{\prime}\left(\tau D_{\tau}+D_{\tau} \tau\right) \check{\psi}} d \sigma d \tau\right\}
$$

We use the rough control (see Corollary 3.6),

$$
\left\|h \partial_{\sigma} \check{\psi}\right\| \leq h^{2 / 3}\|\check{\psi}\|
$$

and the estimates of Agmon to get

$$
h^{2} \operatorname{Re}\left\{\int i \delta^{-1 / 3} \partial_{\sigma}(\sigma \check{\psi}) \overline{\delta^{-4 / 3} \delta^{\prime}\left(\tau D_{\tau}+D_{\tau} \tau\right) \check{\psi}} d \sigma d \tau\right\}=o\left(h^{5 / 3}\right)\|\check{\psi}\|^{2} .
$$

The other terms in the double product can be estimated in the same way so that

$$
\begin{aligned}
& \int\left|\left(i h \delta^{-1 / 3} \partial_{\sigma}+\eta_{0} h^{2 / 3}+\frac{\tau^{2}}{2}+R_{h}-\frac{h \delta^{\prime}}{6 \delta^{4 / 3}}\left(\tau D_{\tau}+D_{\tau} \tau\right)\right) \sigma \check{\psi}\right|^{2} d \sigma d \tau \\
& =\int \mid\left(\left.\left(i h \delta^{-1 / 3} \partial_{\sigma}+\eta_{0} h^{2 / 3}+\frac{\tau^{2}}{2}+\kappa(0) \tau^{3}\right) \sigma \check{\psi}\right|^{2} d \sigma d \tau+o\left(h^{5 / 3}\right)\|\check{\psi}\|^{2} .\right.
\end{aligned}
$$

We can also erase the term in $\tau^{3}$ :

$$
\begin{gathered}
\int\left|\left(i h \delta^{-1 / 3} \partial_{\sigma}+\eta_{0} h^{2 / 3}+\frac{\tau^{2}}{2}+R_{h}-\frac{h \delta^{\prime}}{6 \delta^{4 / 3}}\left(\tau D_{\tau}+D_{\tau} \tau\right)\right) \sigma \check{\psi}\right|^{2} d \sigma d \tau \\
=\int \mid\left(\left.\left(i h \delta^{-1 / 3} \partial_{\sigma}+\eta_{0} h^{2 / 3}+\frac{\tau^{2}}{2}\right) \sigma \check{\psi}\right|^{2} d \sigma d \tau+O\left(h^{5 / 3}\right)\|\check{\psi}\|^{2} .\right.
\end{gathered}
$$

Finally, one will need a last technical detail. We write

$$
\begin{aligned}
& \int\left|\left(i h \delta^{-1 / 3} \partial_{\sigma}+\eta_{0} h^{2 / 3}+\frac{\tau^{2}}{2}\right) \sigma \check{\psi}\right|^{2} d \sigma d \tau \\
& =\int \mid\left(\left.\left(i h \delta^{-1 / 6} \partial_{\sigma} \delta^{-1 / 6}-i h\left(\delta^{-1 / 6}\right)^{\prime}+\eta_{0} h^{2 / 3}+\frac{\tau^{2}}{2}\right) \sigma \breve{\psi}\right|^{2} d \sigma d \tau\right.
\end{aligned}
$$


so that we make the self-adjoint operator $\delta^{-1 / 6} D_{\sigma} \delta^{-1 / 6}$ to appear. We deal with the double products as previously to deduce

$$
\begin{aligned}
& \int\left|\left(i h \delta^{-1 / 6} \partial_{\sigma} \delta^{-1 / 6}-i h\left(\delta^{-1 / 6}\right)^{\prime}+\eta_{0} h^{2 / 3}+\frac{\tau^{2}}{2}\right) \sigma \check{\psi}\right|^{2} d \sigma d \tau \\
& =\int \mid\left(\left.\left(i h \delta^{-1 / 6} \partial_{\sigma} \delta^{-1 / 6}+\eta_{0} h^{2 / 3}+\frac{\tau^{2}}{2}\right) \sigma \check{\psi}\right|^{2} d \sigma d \tau+o\left(h^{5 / 3}\right)\|\breve{\psi}\|^{2} .\right.
\end{aligned}
$$

We have now a nicer lower bound. There exist $c>0$ and $h_{0}>0$ such that, for $h \in\left(0, h_{0}\right)$.

$$
\begin{aligned}
& \check{Q}(\check{\psi}) \\
& \geq \delta_{0}^{2 / 3} v_{1}\left(\eta_{0}\right) h^{4 / 3}\|\check{\psi}\|^{2}+c\left\|h D_{\tau} \sigma \check{\psi}\right\|^{2} \\
& \quad+c \int\left|\left(i h \delta^{-1 / 6} \partial_{\sigma} \delta^{-1 / 6}+\eta_{0} h^{2 / 3}+\frac{\tau^{2}}{2}\right) \sigma \check{\psi}\right|^{2} d \sigma d \tau+O\left(h^{5 / 3}\right)\|\check{\psi}\|^{2} .
\end{aligned}
$$

Using the functional calculus applied to $\delta^{-1 / 6} D_{\sigma} \delta^{-1 / 6}$, we infer that

$$
\breve{Q}(\check{\psi}) \geq \delta_{0}^{2 / 3} v_{1}\left(\eta_{0}\right) h^{4 / 3}\|\breve{\psi}\|^{2}+c v_{1}\left(\eta_{0}\right) h^{4 / 3}\|\sigma \breve{\psi}\|^{2}+O\left(h^{5 / 3}\right)\|\breve{\psi}\|^{2} .
$$

Jointly with the upper bound on $\lambda$, we deduce the result.

Proposition 3.10. There exist $h_{0}>0, C>0$ such that, for $h \in\left(0, h_{0}\right)$ and for all $\check{\psi} \in \mathfrak{E}_{N}(h)$,

$$
\check{Q}(\sigma \check{\psi}) \leq C h^{5 / 3}\|\check{\psi}\|
$$

In particular, we have

$$
\left\|h D_{\tau}(\sigma \check{\psi})\right\|^{2} \leq C h^{5 / 3}\|\breve{\psi}\|^{2} \text { and }\left\|h D_{\sigma}(\sigma \check{\psi})\right\|^{2} \leq C h^{5 / 3}\|\check{\psi}\|^{2} \text {. }
$$

Proof. Let $(\lambda, \psi)$ be an eigenpair of $\mathscr{L}_{h, \mathbf{A}}$. By the IMS formula, we can write

$$
\check{Q}(\sigma \breve{\psi})=\lambda\|\sigma \check{\psi}\|^{2}+\left\|\left[P_{2}(h), \sigma\right] \check{\psi}\right\|^{2}
$$

An immediate computation provides

$$
\left\|\left[P_{2}(h), \sigma\right] \check{\psi}\right\|^{2} \leq C h^{2}\|\check{\psi}\|^{2} .
$$

It remains to use Proposition 3.9.

3.5.2. An improved lower bound. As a consequence of the localization estimates with respect to $\tau$ and of the localization with respect to $\sigma$ given by Proposition 3.9, we have a lower bound for $\check{Q}$. 
Proposition 3.11. There exists $h_{0}>0$ such that for $h \in\left(0, h_{0}\right)$ and $\check{\psi} \in \mathfrak{E}_{N}(h)$

$\check{Q}(\check{\psi})$

$$
\begin{aligned}
\geq \delta_{0}^{2 / 3} \int\left(1+2 k_{0} \tau \delta_{0}^{-1 / 3}\right) \mid & \left(\delta^{-1 / 6} i h \partial_{\sigma} \delta^{-1 / 6}+\eta_{0} h^{2 / 3}\right. \\
& \left.+\frac{\tau^{2}}{2}+\delta_{0}^{-4 / 3} \kappa(0) \tau^{3}\right)\left.\check{\psi}\right|^{2} d \sigma d \tau \\
+\int \delta_{0}^{2 / 3}\left|h D_{\tau} \check{\psi}\right|^{2} d \sigma d \tau & +\frac{2}{3} \delta_{0}^{2 / 3} \alpha \nu_{1}\left(\eta_{0}\right) h^{4 / 3}\|\sigma \check{\psi}\|^{2}+o\left(h^{5 / 3}\right)\|\check{\psi}\|^{2} .
\end{aligned}
$$

Proof. The proof is essentially based on the same estimates as in the proof of Proposition 3.7. Let us recall (3.8)

$$
\begin{aligned}
\check{Q}(\check{\psi}) \geq & \| \check{m}\left(-h D_{\sigma}-\eta_{0}(\delta(\sigma))^{1 / 3} h^{2 / 3}+\check{A}(\sigma, \tau)-h \frac{1}{6} \delta^{\prime} \delta^{-1}\left(\tau D_{\tau}+D_{\tau} \tau\right) \check{\psi} \|^{2}\right. \\
& +\left\|\delta^{1 / 3} h D_{\tau} \check{\psi}\right\|^{2}+o\left(h^{5 / 3}\right)\|\check{\psi}\|^{2} .
\end{aligned}
$$

We transform a little bit (3.9):

$$
\begin{aligned}
\check{Q}(\check{\psi}) \geq & \int \check{m}^{-2}\left|\left(-h D_{\sigma}-\eta_{0} \delta^{1 / 3} h^{2 / 3}+\delta^{1 / 3} \frac{\tau^{2}}{2}+\kappa(0) \delta_{0}^{-1} \tau^{3}\right) \check{\psi}\right|^{2} d \sigma d \tau \\
& +h^{2}\left\|\delta^{1 / 3} D_{\tau} \check{\psi}\right\|^{2}+o\left(h^{5 / 3}\right)\|\check{\psi}\|^{2} .
\end{aligned}
$$

We improve now (3.10) by replacing $\check{m}$ by $1-k_{0} \delta_{0}^{1 / 3} \tau$ thanks to the estimates of Agmon with respect to $\tau$ and using the support of $\breve{\psi}$ with respect to $\sigma$ :

$\check{Q}(\check{\psi})$

$$
\begin{aligned}
\geq & \int\left(1+2 k_{0} \delta_{0}^{1 / 3} \tau\right)\left|\left(-h D_{\sigma}-\eta_{0} \delta^{1 / 3} h^{2 / 3}+\delta^{1 / 3} \frac{\tau^{2}}{2}+\kappa(0) \delta_{0}^{-1} \tau^{3}\right) \check{\psi}\right|^{2} d \sigma d \tau \\
& +h^{2}\left\|\delta^{1 / 3} D_{\tau} \check{\psi}\right\|^{2}+o\left(h^{5 / 3}\right)\|\check{\psi}\|^{2} .
\end{aligned}
$$

It follows

$$
\begin{aligned}
& \check{Q}(\check{\psi}) \\
& \geq \int \delta^{2 / 3}\left(1+2 k_{0} \delta_{0}^{1 / 3} \tau\right) \mid\left(-h \delta^{-1 / 3} D_{\sigma}-\eta_{0} h^{2 / 3}\right. \\
& \left.\quad+\frac{\tau^{2}}{2}+\delta_{0}^{-4 / 3} \kappa(0) \tau^{3}\right)\left.\check{\psi}\right|^{2} d \sigma d \tau \\
& \quad+h^{2}\left\|\delta^{1 / 3} D_{\tau} \check{\psi}\right\|^{2}+o\left(h^{5 / 3}\right)\|\check{\psi}\|^{2} .
\end{aligned}
$$

We use now the Taylor expansion:

$$
\delta^{2 / 3}=\delta_{0}^{2 / 3}\left(1+\frac{2}{3} \alpha \sigma^{2}\right)+O\left(\sigma^{3}\right) .
$$


One of the terms which we can neglect is

$$
\int|\sigma|^{3}\left|h D_{\tau} \breve{\psi}\right|^{2} d \sigma d \tau \leq C h^{2 / 15} \int|\sigma|^{2}\left|h D_{\tau} \check{\psi}\right|^{2} d \sigma d \tau=o\left(h^{5 / 3}\right)\|\breve{\psi}\|^{2},
$$

where we have used Proposition 3.10. In the same way, we can write

$$
\begin{aligned}
\int|\sigma|^{3}\left|\left(1+2 k_{0} \delta_{0}^{1 / 3} \tau\right)\right| \mid( & -h \delta^{-1 / 3} D_{\sigma}-\eta_{0} h^{2 / 3} \\
& \left.+\frac{\tau^{2}}{2}+\delta_{0}^{-4 / 3} \kappa(0) \tau^{3}\right)\left.\check{\psi}\right|^{2} d \sigma d \tau \\
=o\left(h^{5 / 3}\right)\|\check{\psi}\|^{2} . &
\end{aligned}
$$

It remains to analyze

$$
\frac{2 \alpha}{3} \delta_{0}^{2 / 3} \int \sigma^{2}\left|\left(-h \delta^{-1 / 3} D_{\sigma}-\eta_{0} h^{2 / 3}+\frac{\tau^{2}}{2}+\delta_{0}^{-4 / 3} \kappa(0) \tau^{3}\right) \check{\psi}\right|^{2} d \sigma d \tau .
$$

This can be done in the same way as in the proof of Proposition 3.9, see (3.15):

$$
\begin{aligned}
& \frac{2 \alpha}{3} \delta_{0}^{2 / 3} \int \sigma^{2}\left|\left(-h \delta^{-1 / 3} D_{\sigma}-\eta_{0} h^{2 / 3}+\frac{\tau^{2}}{2}+\delta_{0}^{-4 / 3} \kappa(0) \tau^{3}\right) \check{\psi}\right|^{2} d \sigma d \tau \\
& \quad=\frac{2 \alpha}{3} \delta_{0}^{2 / 3} \int\left|\left(-h \delta^{-1 / 3} D_{\sigma}-\eta_{0} h^{2 / 3}+\frac{\tau^{2}}{2}\right) \sigma \check{\psi}\right|^{2} d \sigma d \tau+o\left(h^{5 / 3}\right)\|\check{\psi}\|^{2} .
\end{aligned}
$$

We deduce

$$
\begin{aligned}
\check{Q}(\check{\psi}) \geq \int \delta_{0}^{2 / 3}\left(\left|h D_{\tau} \check{\psi}\right|^{2}+\left(1+2 k_{0} \tau \delta_{0}^{-1 / 3}\right) \mid\left(\delta^{-1 / 3} i h \partial_{\sigma}+\eta_{0} h^{2 / 3}\right.\right. \\
\left.\left.+\frac{\tau^{2}}{2}+\delta_{0}^{-4 / 3} \kappa(0) \tau^{3}\right)\left.\check{\psi}\right|^{2}\right) d \sigma d \tau \\
+\frac{2}{3} \delta_{0}^{2 / 3} \alpha \nu_{1}\left(\eta_{0}\right) h^{4 / 3}\|\sigma \check{\psi}\|^{2}+o\left(h^{5 / 3}\right)\|\check{\psi}\|^{2} .
\end{aligned}
$$

We replace $\delta^{-1 / 3} \partial_{\sigma}$ by $\delta^{-1 / 6} \partial_{\sigma} \delta^{-1 / 6}$ as in (3.16) and the conclusion follows.

3.5.3. Localization with respect to $\boldsymbol{D}_{\boldsymbol{\sigma}}$. In this subsection, we investigate a microlocalization property with respect to $D_{\sigma}$.

Proposition 3.12. There exist $h_{0}>0, C>0$ such that, for $h \in\left(0, h_{0}\right)$ and for all $\check{\psi} \in \mathfrak{E}_{N}(h)$,

$$
\left\|D_{\sigma} \breve{\psi}\right\| \leq C h^{-1 / 6}\|\check{\psi}\|
$$

We introduce a new coordinate

$$
\varsigma=f(\sigma)=\int_{0}^{\sigma} \delta(\sigma)^{1 / 3} d \sigma
$$


The space $L^{2}(d \sigma d \tau)$ becomes $L^{2}\left(\delta^{-1 / 3} d \varsigma d \tau\right)$. We can write

$$
\partial_{\sigma}=\delta^{1 / 3}(\sigma) \partial_{\varsigma}
$$

Notation 3.13. We will denote by $\check{\varphi}$ the function $\breve{\psi}$ in the coordinates $(\varsigma, \tau)$.

Fourier transform. We are led to introduce a weighted Fourier transform

$$
\mathcal{F}_{\delta}(\check{\varphi})(\mu)=\mathscr{F}\left(\delta^{-1 / 6} \check{\varphi}\right) \text {. }
$$

A straight forward computation provides

$$
\mathcal{F}_{\delta}\left(\left(\delta^{1 / 6} i h \partial_{\varsigma} \delta^{-1 / 6}\right) \check{\varphi}\right)(\mu)=-\mu \mathcal{F}_{\delta}(\check{\varphi})(\mu)
$$

and

$$
D_{\mu} \widetilde{F}_{\delta}(\check{\varphi})(\mu)=-\mathcal{F}_{\delta}(\varsigma \check{\varphi})(\mu) .
$$

Using the Parseval formula, we see that $\mathcal{F}_{\delta}$ is unitary from $L^{2}\left(\delta^{-1 / 3} d \varsigma d \tau\right)$ to $L^{2}(d \mu d \tau)$. We can now prove a microlocal estimate with respect to $\delta^{-1 / 6} D_{\sigma} \delta^{-1 / 6}$ which implies Proposition 3.12.

Proposition 3.14. There exist $h_{0}>0, C>0$ such that, for $h \in\left(0, h_{0}\right)$ and for all $\check{\psi} \in \mathfrak{E}_{N}(h)$,

$$
\left\|\delta^{1 / 6} D_{\varsigma} \delta^{-1 / 6} \check{\varphi}\right\|_{L^{2}\left(\delta^{-1 / 3} d \varsigma d \tau\right)} \leq C h^{-1 / 6}\|\breve{\psi}\|
$$

To prove the proposition we need the following lemma.

\section{Lemma 3.15. We have}

$$
\left\|h D_{\tau}\left(\left(\delta^{-1 / 6} \partial_{\sigma} \delta^{-1 / 6}\right) \check{\psi}\right)\right\|^{2} \leq C h^{4 / 3}\left\|\left(\delta^{-1 / 6} \partial_{\sigma} \delta^{-1 / 6}\right) \check{\psi}\right\|^{2}+C h^{4 / 3}\|\check{\psi}\|^{2}
$$

and

$$
\left\|h D_{\sigma}\left(\left(\delta^{-1 / 6} \partial_{\sigma} \delta^{-1 / 6}\right) \breve{\psi}\right)\right\|^{2} \leq C h^{4 / 3}\left\|\left(\delta^{-1 / 6} \partial_{\sigma} \delta^{-1 / 6}\right) \breve{\psi}\right\|^{2}+C h^{4 / 3}\|\breve{\psi}\|^{2} .
$$

Proof. Let $(\lambda, \psi)$ be an eigenpair of $\mathscr{L}_{h, \mathbf{A}}$. We use the IMS formula and we get

$$
\begin{aligned}
\breve{Q}\left(\left(\delta^{-1 / 6} \partial_{\sigma} \delta^{-1 / 6}\right) \check{\psi}\right) \\
=\lambda\left\|\left(\delta^{-1 / 6} \partial_{\sigma} \delta^{-1 / 6}\right) \check{\psi}\right\|^{2}-\left\|\left[P_{1}(h),\left(\delta^{-1 / 6} \partial_{\sigma} \delta^{-1 / 6}\right)\right] \breve{\psi}\right\|^{2} \\
\quad-\left\|\left[P_{2}(h),\left(\delta^{-1 / 6} \partial_{\sigma} \delta^{-1 / 6}\right)\right] \breve{\psi}\right\|^{2}+O\left(h^{2}\right)\|\breve{\psi}\|^{2} .
\end{aligned}
$$

A computation of the commutators provides

$$
\left\|\left[P_{1}(h),\left(\delta^{-1 / 6} \partial_{\sigma} \delta^{-1 / 6}\right)\right] \check{\psi}\right\|^{2} \leq C h^{4 / 3}\|\check{\psi}\|^{2}
$$


and

$$
\left\|\left[P_{2}(h),\left(\delta^{-1 / 6} \partial_{\sigma} \delta^{-1 / 6}\right)\right] \breve{\psi}\right\|^{2} \leq C h^{4 / 3}\|\check{\psi}\|^{2} .
$$

This implies

$$
\check{Q}\left(\left(\delta^{-1 / 6} \partial_{\sigma} \delta^{-1 / 6}\right) \check{\psi}\right) \leq \lambda\left\|\left(\delta^{-1 / 6} \partial_{\sigma} \delta^{-1 / 6}\right) \check{\psi}\right\|^{2}+C h^{4 / 3}\|\breve{\psi}\|^{2} .
$$

We immediately deduce (3.18). For the proof of (3.19), we write

$$
\left\|P_{1}(h)\left(\delta^{-1 / 6} \partial_{\sigma} \delta^{-1 / 6}\right) \breve{\psi}\right\|^{2} \leq \lambda\left\|\left(\delta^{-1 / 6} \partial_{\sigma} \delta^{-1 / 6}\right) \breve{\psi}\right\|^{2}+C h^{4 / 3}\|\breve{\psi}\|^{2} .
$$

It remains to investigate the sizes of the different terms appearing in $P_{1}(h)$. We observe (see Corollary 3.6) that

$$
\left\|\tau^{2}\left(\delta^{-1 / 6} \partial_{\sigma} \delta^{-1 / 6}\right) \check{\psi}\right\|^{2} \leq C h^{4 / 3}\left\|\left(\delta^{-1 / 6} \partial_{\sigma} \delta^{-1 / 6}\right) \check{\psi}\right\|^{2}+C h^{4 / 3}\|\breve{\psi}\|^{2} .
$$

and

$$
\begin{aligned}
& \left\|h \delta^{\prime}\left(\tau D_{\tau}+D_{\tau} \tau\right)\left(\delta^{-1 / 6} \partial_{\sigma} \delta^{-1 / 6}\right) \check{\psi}\right\|^{2} \\
& \quad \leq C\left\|h D_{\tau}\left(\delta^{-1 / 6} \partial_{\sigma} \delta^{-1 / 6}\right) \check{\psi}\right\|^{2}+C\left\|h \delta^{\prime}\left(\delta^{-1 / 6} \partial_{\sigma} \delta^{-1 / 6}\right) \check{\psi}\right\|^{2} \\
& \quad \leq C h^{4 / 3}\left\|\left(\delta^{-1 / 6} \partial_{\sigma} \delta^{-1 / 6}\right) \check{\psi}\right\|^{2}+C h^{4 / 3}\|\check{\psi}\|^{2},
\end{aligned}
$$

where we have used (3.18).

Proof of Proposition 3.14. Let $(\lambda, \psi)$ be an eigenpair of $\mathscr{L}_{h, \mathbf{A}}$.

Microlocal estimate near the minimum. We have (see Proposition 3.11 jointly with Corollary 3.6)

$$
\begin{aligned}
\check{Q}(\breve{\psi}) \geq \int & \delta_{0}^{2 / 3}\left(\left|h D_{\tau} \breve{\psi}\right|^{2}+\left|\left(\delta^{-1 / 6} i h \partial_{\sigma} \delta^{-1 / 6}+\eta_{0} h^{2 / 3}+\frac{\tau^{2}}{2}\right) \check{\psi}\right|^{2}\right) d \sigma d \tau \\
& -C h^{5 / 3}\|\check{\psi}\|^{2} .
\end{aligned}
$$

This becomes

$$
\begin{aligned}
\check{Q}(\check{\psi}) \geq \int & \delta_{0}^{2 / 3}\left(\left|h D_{\tau} \check{\psi}\right|^{2}+\left|\left(-h \mu+\eta_{0} h^{2 / 3}+\frac{\tau^{2}}{2}\right) \mathcal{F}_{\delta}(\check{\varphi})\right|^{2}\right) d \mu d \tau \\
& -C h^{5 / 3}\|\check{\psi}\|^{2} .
\end{aligned}
$$

We infer

$$
\check{Q}(\check{\psi}) \geq \int h^{4 / 3} \delta_{0}^{2 / 3} v_{1}\left(\eta_{0}-h^{1 / 3} \mu\right)\left|\widetilde{F}_{\delta}(\check{\varphi})\right|^{2} d \mu d \tau-C h^{5 / 3}\|\check{\psi}\|^{2} .
$$

Let us fix $\varepsilon_{0}>0$ small enough to have, for $\left|h^{1 / 3} \mu\right| \leq \varepsilon_{0}$,

$$
v_{1}\left(\eta_{0}-h^{1 / 3} \mu\right) \geq v_{1}\left(\eta_{0}\right)+\frac{v^{\prime \prime}\left(\eta_{0}\right)}{4} h^{2 / 3} \mu^{2} .
$$


For this value of $\varepsilon_{0}$, there exists $c_{0}>0$ such that, for $\left|h^{1 / 3} \mu\right| \geq \varepsilon_{0}$

$$
v_{1}\left(\eta_{0}-h^{1 / 3} \mu\right) \geq v_{1}\left(\eta_{0}\right)+c_{0} .
$$

Let us recall that $\check{Q}(\check{\psi}) \leq\left(\lambda+O\left(h^{\infty}\right)\right)\|\check{\psi}\|^{2}$ and that $\lambda$ satisfies (3.11). Then, it remains to split the integrals on $\left|h^{1 / 3} \mu\right| \leq \varepsilon_{0}$ and $\left|h^{1 / 3} \mu\right| \geq \varepsilon_{0}$ and we infer

$$
\int_{\left|h^{1 / 3} \mu\right| \geq \varepsilon_{0}}\left|\mathscr{F}_{\delta}(\check{\varphi})\right|^{2} d \mu d \tau \leq C h^{1 / 3}\|\check{\psi}\|^{2},
$$

and

$$
\int_{\left|h^{1 / 3} \mu\right| \leq \varepsilon_{0}}\left|\mu \mathcal{F}_{\delta}(\check{\varphi})\right|^{2} d \mu d \tau \leq C h^{-1 / 3}\|\check{\psi}\|^{2} .
$$

Microlocal estimate away from the minimum. Then, we want to obtain a control of

$$
\int_{\left|h^{1 / 3} \mu\right| \geq \varepsilon_{0}}\left|\mu \mathcal{F}_{\delta}(\check{\varphi})\right|^{2} d \mu d \tau .
$$

For that purpose, we will use Lemma 3.15. Let us first write

$$
\begin{aligned}
& \check{Q}\left(\left(\delta^{-1 / 6} \partial_{\sigma} \delta^{-1 / 6}\right) \check{\psi}\right) \\
& \quad \geq\left\|P_{1}(h)\left(\delta^{-1 / 6} \partial_{\sigma} \delta^{-1 / 6}\right) \check{\psi}\right\|^{2}+h^{2}\left\|\delta^{1 / 3} D_{\tau}\left(\delta^{-1 / 6} \partial_{\sigma} \delta^{-1 / 6}\right) \check{\psi}\right\|^{2} \\
& \quad-C h^{2}\left\|\left(\delta^{-1 / 6} \partial_{\sigma} \delta^{-1 / 6}\right) \check{\psi}\right\|^{2} .
\end{aligned}
$$

We have immediately

$$
h^{2}\left\|\delta^{1 / 3} D_{\tau}\left(\delta^{-1 / 6} \partial_{\sigma} \delta^{-1 / 6}\right) \check{\psi}\right\|^{2} \geq \delta_{0}^{2 / 3} h^{2}\left\|D_{\tau}\left(\delta^{-1 / 6} \partial_{\sigma} \delta^{-1 / 6}\right) \check{\psi}\right\|^{2} .
$$

Then, we write

$$
\left\|P_{1}(h)\left(\delta^{-1 / 6} \partial_{\sigma} \delta^{-1 / 6}\right) \check{\psi}\right\|^{2} \geq\left\|\left(\check{m}^{-1} P+C_{h}\right)\left(\delta^{-1 / 6} \partial_{\sigma} \delta^{-1 / 6}\right) \check{\psi}\right\|^{2} .
$$

where

$$
P=-h D_{\sigma}-\eta_{0}(\delta(\sigma))^{1 / 3} h^{2 / 3}+\check{A}(\sigma, \tau)-\frac{h}{6} \delta^{\prime} \delta^{-1}\left(\tau D_{\tau}+D_{\tau} \tau\right) .
$$

Expanding the square, we are led to estimate the following term:

$$
\operatorname{Re}\left\langle\check{m}^{-1} P\left(\delta^{-1 / 6} \partial_{\sigma} \delta^{-1 / 6}\right) \check{\psi}, C_{h}\left(\delta^{-1 / 6} \partial_{\sigma} \delta^{-1 / 6}\right) \check{\psi}\right\rangle
$$

We have

$$
\left\|C_{h}\left(\delta^{-1 / 6} \partial_{\sigma} \delta^{-1 / 6}\right) \check{\psi}\right\| \leq C h\left\|\left(\delta^{-1 / 6} \partial_{\sigma} \delta^{-1 / 6}\right) \check{\psi}\right\|
$$

and

$$
\left\|\check{m}^{-1} P\left(\delta^{-1 / 6} \partial_{\sigma} \delta^{-1 / 6}\right) \check{\psi}\right\| \leq C\left\|P\left(\delta^{-1 / 6} \partial_{\sigma} \delta^{-1 / 6}\right) \check{\psi}\right\| .
$$


We get

$$
\left\|P\left(\delta^{-1 / 6} \partial_{\sigma} \delta^{-1 / 6}\right) \breve{\psi}\right\| \leq C h\left\|\delta^{\prime}\left(\tau D_{\tau}+D_{\tau} \tau\right)\left(\delta^{-1 / 6} \partial_{\sigma} \delta^{-1 / 6}\right) \breve{\psi}\right\|
$$

and

$$
\begin{aligned}
& \left\|\delta^{\prime}\left(\tau D_{\tau}+D_{\tau} \tau\right)\left(\delta^{-1 / 6} \partial_{\sigma} \delta^{-1 / 6}\right) \check{\psi}\right\| \\
& \quad \leq C h\left\|\left(\delta^{-1 / 6} \partial_{\sigma} \delta^{-1 / 6}\right) \check{\psi}\right\|+C\left\|\delta^{\prime} \tau D_{\tau}\left(\delta^{-1 / 6} \partial_{\sigma} \delta^{-1 / 6}\right) \check{\psi}\right\| .
\end{aligned}
$$

Using the support of $\breve{\psi}$, we deduce

$$
\left\|\delta^{\prime} \tau D_{\tau}\left(\delta^{-1 / 6} \partial_{\sigma} \delta^{-1 / 6}\right) \breve{\psi}\right\| \leq C h^{1 / 3}\left\|D_{\tau}\left(\delta^{-1 / 6} \partial_{\sigma} \delta^{-1 / 6}\right) \breve{\psi}\right\| .
$$

Therefore, with (3.18), we infer

$$
\begin{aligned}
& \left\|P_{1}(h)\left(\delta^{-1 / 6} \partial_{\sigma} \delta^{-1 / 6}\right) \check{\psi}\right\|^{2} \\
& \quad \geq\left\|\left(\check{m}^{-1} P\right)\left(\delta^{-1 / 6} \partial_{\sigma} \delta^{-1 / 6}\right) \check{\psi}\right\|^{2}-C h^{5 / 3}\left\|\left(\delta^{-1 / 6} \partial_{\sigma} \delta^{-1 / 6}\right) \check{\psi}\right\|^{2}-C h^{5 / 3}\|\check{\psi}\|^{2} .
\end{aligned}
$$

We write

$$
\left\|\left(\check{m}^{-1} P\right)\left(\delta^{-1 / 6} \partial_{\sigma} \delta^{-1 / 6}\right) \check{\psi}\right\|^{2} \geq \delta_{0}^{2 / 3}\left\|\left(\check{m}^{-1} \delta^{-1 / 3} P\right)\left(\delta^{-1 / 6} \partial_{\sigma} \delta^{-1 / 6}\right) \check{\psi}\right\|^{2} .
$$

We shall again expand the square and control the term

$$
\left\|\tau^{2}\left(\delta^{-1 / 6} \partial_{\sigma} \delta^{-1 / 6}\right) \check{\psi}\right\| \leq C h^{2 / 3}\left(\left\|\left(\delta^{-1 / 6} \partial_{\sigma} \delta^{-1 / 6}\right) \check{\psi}\right\|+\|\check{\psi}\|\right) .
$$

With (3.21), (3.22), (3.18) and (3.19), it follows that

$$
\begin{aligned}
& \left\|P_{1}(h)\left(\delta^{-1 / 6} \partial_{\sigma} \delta^{-1 / 6}\right) \check{\psi}\right\|^{2} \\
& \geq \delta_{0}^{2 / 3}\left\|\check{m}^{-1}\left(-h \delta^{-1 / 3} D_{\sigma}-\eta_{0} h^{2 / 3}+\frac{\check{A}}{\delta^{1 / 3}}\right)\left(\delta^{-1 / 6} \partial_{\sigma} \delta^{-1 / 6}\right) \check{\psi}\right\|^{2} \\
& \quad-C h^{5 / 3}\left\|\left(\delta^{-1 / 6} \partial_{\sigma} \delta^{-1 / 6}\right) \check{\psi}\right\|^{2}-C h^{5 / 3}\|\check{\psi}\|^{2} .
\end{aligned}
$$

With the same arguments and using the Taylor expansion of $\breve{A}$, we find

$$
\begin{aligned}
& \left\|P_{1}(h)\left(\delta^{-1 / 6} \partial_{\sigma} \delta^{-1 / 6}\right) \breve{\psi}\right\|^{2} \\
& \geq \delta_{0}^{2 / 3}\left\|\check{m}^{-1}\left(-h \delta^{-1 / 3} D_{\sigma}-\eta_{0} h^{2 / 3}+\frac{\tau^{2}}{2}\right)\left(\delta^{-1 / 6} \partial_{\sigma} \delta^{-1 / 6}\right) \check{\psi}\right\|^{2} \\
& \quad-C h^{5 / 3}\left\|\left(\delta^{-1 / 6} \partial_{\sigma} \delta^{-1 / 6}\right) \check{\psi}\right\|^{2}-C h^{5 / 3}\|\check{\psi}\|^{2} .
\end{aligned}
$$

Let us notice that, see (3.19),

$$
\begin{aligned}
& \int|\tau|\left|\left(-h \delta^{-1 / 3} D_{\sigma}-\eta_{0} h^{2 / 3}+\frac{\tau^{2}}{2}\right)\left(\delta^{-1 / 6} \partial_{\sigma} \delta^{-1 / 6}\right) \check{\psi}\right|^{2} d \sigma d \tau \\
& \leq C h^{1 / 3-\gamma} \int\left|\left(-h \delta^{-1 / 3} D_{\sigma}-\eta_{0} h^{2 / 3}+\frac{\tau^{2}}{2}\right)\left(\delta^{-1 / 6} \partial_{\sigma} \delta^{-1 / 6}\right) \check{\psi}\right|^{2} d \sigma d \tau \\
& \leq C h^{5 / 3-\gamma}\left\|\left(\delta^{-1 / 6} \partial_{\sigma} \delta^{-1 / 6}\right) \check{\psi}\right\|^{2}+C h^{5 / 3-\gamma}\|\check{\psi}\|^{2} .
\end{aligned}
$$


Using the Taylor expansion of $\check{m}$ and (3.23), we find

$$
\begin{aligned}
& \check{Q}\left(\left(\delta^{-1 / 6} \partial_{\sigma} \delta^{-1 / 6}\right) \check{\psi}\right) \\
& \geq \int \delta_{0}^{2 / 3}\left(\left|h D_{\tau} \check{\psi}\right|^{2}\right. \\
& \left.\quad+\left|\left(\delta^{-1 / 3} i h \partial_{\sigma}+\eta_{0} h^{2 / 3}+\frac{\tau^{2}}{2}\right)\left(\delta^{-1 / 6} \partial_{\sigma} \delta^{-1 / 6}\right) \check{\psi}\right|^{2}\right) d \sigma d \tau \\
& \quad-C h^{5 / 3-\gamma}\left\|\left(\delta^{-1 / 6} \partial_{\sigma} \delta^{-1 / 6}\right) \check{\psi}\right\|^{2}-C h^{5 / 3-\gamma}\|\check{\psi}\|^{2} .
\end{aligned}
$$

Replacing $\delta^{-1 / 3} \partial_{\sigma}$ by $\delta^{-1 / 6} \partial_{\sigma} \delta^{-1 / 6}$ (modulo error terms which can be controlled with the same arguments), we deduce

$$
\begin{aligned}
& \check{Q}\left(\left(\delta^{-1 / 6} \partial_{\sigma} \delta^{-1 / 6}\right) \check{\psi}\right) \\
& \geq \int \delta_{0}^{2 / 3}\left(\left|h D_{\tau} \check{\psi}\right|^{2}\right. \\
& \left.+\left|\left(\delta^{-1 / 6} i h \partial_{\sigma} \delta^{-1 / 6}+\eta_{0} h^{2 / 3}+\frac{\tau^{2}}{2}\right)\left(\delta^{-1 / 6} \partial_{\sigma} \delta^{-1 / 6}\right) \check{\psi}\right|^{2}\right) d \sigma d \tau \\
& -C h^{5 / 3-\gamma}\left\|\left(\delta^{-1 / 6} \partial_{\sigma} \delta^{-1 / 6}\right) \check{\psi}\right\|^{2}-C h^{5 / 3-\gamma}\|\check{\psi}\|^{2} \text {. }
\end{aligned}
$$

We infer

$$
\begin{aligned}
\check{Q}(\check{\psi}) \geq \int & h^{4 / 3} \delta_{0}^{2 / 3} v_{1}\left(\eta_{0}-h^{1 / 3} \mu\right)\left|\mu \widetilde{F}_{\delta}(\check{\varphi})\right|^{2} d \mu d \tau \\
& -C h^{5 / 3-\gamma}\left\|\left(\delta^{-1 / 6} \partial_{\sigma} \delta^{-1 / 6}\right) \check{\psi}\right\|^{2}-C h^{5 / 3-\gamma}\|\check{\psi}\|^{2} .
\end{aligned}
$$

It follows

$$
\left.\int\right|_{h^{1 / 3} \mu \mid \geq \varepsilon_{0}}\left|\mu \mathcal{F}_{\delta} \check{\varphi}\right|^{2} d \mu d \tau \leq C h^{1 / 3-\gamma}\left\|\mu \mathcal{F}_{\delta} \check{\varphi}\right\|^{2}+C h^{1 / 3-\gamma}\|\check{\psi}\|^{2} .
$$

Combining this last estimate with (3.20), we get the conclusion.

\section{Approximation by tensor products}

We can now prove an approximation result for the eigenfunctions. Let us recall the rescaled coordinates (see (2.3)):

$$
\sigma=h^{1 / 6} \hat{\sigma} \quad \text { and } \quad \tau=h^{1 / 3} \hat{\tau} .
$$

Notation 4.1. $\widehat{\mathscr{L}}(h)$ denotes $h^{-4 / 3} \check{\mathscr{L}}(h)$ in the coordinates $(\hat{\sigma}, \hat{\tau})$. The corresponding quadratic form will be denoted by $\hat{Q}$. We will use the notation $\hat{\mathfrak{E}}_{N}(h)$ to denote $\mathfrak{E}_{N}(h)$ after rescaling. 
We introduce the Feshbach-Grushin projection

$$
\Pi_{0} \varphi=\left\langle\varphi, u_{\eta_{0}}\right\rangle_{\hat{\tau}} u_{\eta_{0}}(\hat{\tau})
$$

We will need to consider the quadratic form

$$
\hat{Q}_{0}(\varphi)=\delta_{0}^{2 / 3} \int\left|D_{\hat{\tau}} \varphi\right|^{2}+\left|\left(-\eta_{0}+\frac{\hat{\tau}^{2}}{2}\right) \varphi\right|^{2} d \hat{\sigma} d \hat{\tau} .
$$

Proposition 4.2. There exist $h_{0}>0$ and $C>0$ such that for $h \in\left(0, h_{0}\right)$ and $\hat{\psi} \in \widehat{\mathfrak{E}}_{N}(h):$

$$
0 \leq \hat{Q}_{0}(\hat{\psi})-\delta_{0}^{2 / 3} v_{1}\left(\eta_{0}\right)\|\hat{\psi}\|^{2} \leq C h^{1 / 6}\|\hat{\psi}\|^{2}
$$

and

$$
\begin{aligned}
\left\|\Pi_{0} \hat{\psi}-\hat{\psi}\right\| & \leq C h^{1 / 12}\|\hat{\psi}\|, \\
\left\|D_{\hat{\tau}}\left(\Pi_{0} \hat{\psi}-\hat{\psi}\right)\right\| & \leq C h^{1 / 12}\|\hat{\psi}\|, \\
\left\|\hat{\tau}^{2}\left(\Pi_{0} \hat{\psi}-\hat{\psi}\right)\right\| & \leq C h^{1 / 12}\|\hat{\psi}\| .
\end{aligned}
$$

Proof. Let us consider $\hat{\psi} \in \hat{\mathfrak{E}}_{N}(h)$ which is associated with a rescaled eigenvalue $\hat{\lambda}$. We have

$$
\hat{Q}(\hat{\psi}) \leq\left(\hat{\lambda}+O\left(h^{\infty}\right)\right)\|\hat{\psi}\|^{2}
$$

and

$$
\left\|\widehat{P}_{1}(h) \hat{\psi}\right\|^{2}+\left\|\widehat{P}_{2}(h) \hat{\psi}\right\|^{2} \leq\left(v_{1}\left(\eta_{0}\right)+C h^{1 / 3}\right)\|\hat{\psi}\|^{2} .
$$

We now use Proposition 3.11 to get (the term in $\tau^{3}$ and the term associated with $\check{m}$ are controlled by the estimates of Agmon)

$$
\begin{aligned}
& \hat{Q}(\hat{\psi}) \\
& \geq \int \delta_{0}^{2 / 3}\left(\left|D_{\hat{\tau}} \hat{\psi}\right|^{2}\right. \\
& \left.+\left|\left(\hat{\delta}^{-1 / 6} i h^{1 / 6} \partial_{\hat{\sigma}} \hat{\delta}^{-1 / 6}-\eta_{0}+\frac{\hat{\tau}^{2}}{2}\right) \hat{\psi}\right|^{2}\right) d \hat{\sigma} d \hat{\tau}+o\left(h^{1 / 3}\right)\|\hat{\psi}\|^{2} .
\end{aligned}
$$

We wish to make the term $\hat{\delta}^{-1 / 6} i h^{1 / 6} \partial_{\hat{\sigma}} \hat{\delta}^{-1 / 6}$ to disappear modulo some error term. Expanding the square, we are led to estimate the double product

$$
2 \operatorname{Re}\left\langle\left(-\eta_{0}+\frac{\hat{\tau}^{2}}{2}\right) \hat{\psi}, \hat{\delta}^{-1 / 6} i h^{1 / 6} \partial_{\hat{\sigma}} \hat{\delta}^{-1 / 6} \hat{\psi}\right\rangle .
$$

We have

$$
\left\|\left(-\eta_{0}+\frac{\hat{\tau}^{2}}{2}\right) \hat{\psi}\right\| \leq C\|\hat{\psi}\|
$$


and, with Proposition 3.12,

$$
\left\|\hat{\delta}^{-1 / 6} i h^{1 / 6} \partial_{\hat{\sigma}} \hat{\delta}^{-1 / 6} \hat{\psi}\right\| \leq C h^{1 / 6}\left\|\partial_{\hat{\sigma}} \hat{\delta}^{-1 / 6} \hat{\psi}\right\| \leq C h^{1 / 6}\|\hat{\psi}\|
$$

It follows

$$
\hat{Q}(\hat{\psi}) \geq \hat{Q}_{0}(\hat{\psi})-C h^{1 / 6}\|\hat{\psi}\|^{2}
$$

We deduce (4.2). We get (4.3) as a consequence of (4.2) in a standard way (using that the second eigenvalue of $\widehat{Q}_{0}$ is strictly larger than the first one).

Proposition 4.3. There exist $h_{0}>0$ and $C>0$ such that for $h \in\left(0, h_{0}\right)$ and $\hat{\psi} \in \hat{\mathfrak{E}}_{N}(h):$

$$
\begin{gathered}
\hat{Q}(\hat{\psi}) \geq \int \delta_{0}^{2 / 3}\left(\left|D_{\hat{\tau}} \hat{\psi}\right|^{2}+\left|\left(\hat{\delta}^{-1 / 6} i h^{1 / 6} \partial_{\hat{\sigma}} \hat{\delta}^{-1 / 6}-\eta_{0}+\frac{\hat{\tau}^{2}}{2}\right) \hat{\psi}\right|^{2}\right) d \hat{\sigma} d \hat{\tau} \\
+\frac{2}{3} \delta_{0}^{2 / 3} \alpha v_{1}\left(\eta_{0}\right)\|\hat{\sigma} \hat{\psi}\|^{2}+C_{0} h^{1 / 3}\|\hat{\psi}\|^{2}+o\left(h^{1 / 3}\right)\|\hat{\psi}\|^{2},
\end{gathered}
$$

where $C_{0}$ is defined in (1.10).

Proof. We use Proposition 3.11 to write

$$
\begin{gathered}
\hat{Q}(\hat{\psi}) \begin{array}{c}
\delta_{0}^{2 / 3} \int\left(\left|h D_{\hat{\tau}} \hat{\psi}\right|^{2}\right. \\
+\mid\left(\hat{\delta}^{-1 / 6} i h^{1 / 6} \partial_{\hat{\sigma}} \hat{\delta}^{-1 / 6}-\eta_{0}\right. \\
\left.\left.+\frac{\hat{\tau}^{2}}{2}+\delta_{0}^{-4 / 3} \kappa(0) h^{1 / 3} \hat{\tau}^{3}\right)\left.\hat{\psi}\right|^{2}\right) d \hat{\sigma} d \hat{\tau}
\end{array} \\
+2 h^{1 / 3} k_{0} \delta_{0}^{-1 / 3} \int \hat{\tau} \mid\left(\hat{\delta}^{-1 / 6} i h^{1 / 6} \partial_{\hat{\sigma}} \hat{\delta}^{-1 / 6}-\eta_{0}\right. \\
\left.+\frac{\hat{\tau}^{2}}{2}+\delta_{0}^{-4 / 3} \kappa(0) h^{1 / 3} \hat{\tau}^{3}\right)\left.\hat{\psi}\right|^{2} d \hat{\sigma} d \tau \\
+\frac{2}{3} \delta_{0}^{2 / 3} \alpha \nu_{1}\left(\eta_{0}\right)\|\hat{\sigma} \hat{\psi}\|^{2}+o\left(h^{1 / 3}\right)\|\hat{\psi}\|^{2} .
\end{gathered}
$$

With the estimates of Agmon and Proposition 3.12, we get on the one hand

$$
\begin{gathered}
\int \hat{\tau}\left|\left(\hat{\delta}^{-1 / 6} i h^{1 / 6} \partial_{\hat{\sigma}} \hat{\delta}^{-1 / 6}-\eta_{0}+\frac{\hat{\tau}^{2}}{2}+\delta_{0}^{-4 / 3} \kappa(0) h^{1 / 3} \hat{\tau}^{3}\right) \hat{\psi}\right|^{2} d \hat{\sigma} d \tau \\
=h^{1 / 3} \int \hat{\tau}\left(-\eta_{0}+\frac{\hat{\tau}^{2}}{2}\right)^{2}|\hat{\psi}|^{2} d \hat{\sigma} d \hat{\tau}+o\left(h^{1 / 3}\right)\|\hat{\psi}\|^{2}
\end{gathered}
$$


and on the other hand

$$
\begin{aligned}
\int \mid & \left.\left(\hat{\delta}^{-1 / 6} i h^{1 / 6} \partial_{\hat{\sigma}} \hat{\delta}^{-1 / 6}-\eta_{0}+\frac{\hat{\tau}^{2}}{2}+\delta_{0}^{-4 / 3} \kappa(0) h^{1 / 3} \hat{\tau}^{3}\right) \hat{\psi}\right|^{2} d \hat{\sigma} d \hat{\tau} \\
= & \int\left|\left(\hat{\delta}^{-1 / 6} i h^{1 / 6} \partial_{\hat{\sigma}} \hat{\delta}^{-1 / 6}-\eta_{0}+\frac{\hat{\tau}^{2}}{2}\right) \hat{\psi}\right|^{2} d \hat{\sigma} d \hat{\tau} \\
& +2 \delta_{0}^{-4 / 3} \kappa(0) h^{1 / 3} \int\left(-\eta_{0}+\frac{\hat{\tau}^{2}}{2}\right) \hat{\tau}^{3}|\hat{\psi}|^{2} \hat{\sigma} d \tau+o\left(h^{1 / 3}\right)\|\hat{\psi}\|^{2} .
\end{aligned}
$$

It remains to approximate the quantities

$$
\int \hat{\tau}\left(-\eta_{0}+\frac{\hat{\tau}^{2}}{2}\right)^{2}|\hat{\psi}|^{2} d \hat{\sigma} d \hat{\tau}
$$

and

$$
\int\left(-\eta_{0}+\frac{\hat{\tau}^{2}}{2}\right) \hat{\tau}^{3}|\hat{\psi}|^{2} \hat{\sigma} d \tau
$$

Let us analyze the first one. We consider

$$
\begin{aligned}
& \left|\int \hat{\tau}\left(-\eta_{0}+\frac{\hat{\tau}^{2}}{2}\right)^{2}\left(|\hat{\psi}|^{2}-\left|\Pi_{0} \hat{\psi}\right|^{2}\right) d \hat{\sigma} d \hat{\tau}\right| \\
& \quad \leq\left|\int \hat{\tau}\left(-\eta_{0}+\frac{\hat{\tau}^{2}}{2}\right)^{2}\left(|\hat{\psi}|-\left|\Pi_{0} \hat{\psi}\right|\right)\left(|\hat{\psi}|+\left|\Pi_{0} \hat{\psi}\right|\right) d \hat{\sigma} d \hat{\tau}\right| \\
& \quad \leq \int\left|\hat{\tau}\left(-\eta_{0}+\frac{\hat{\tau}^{2}}{2}\right)^{2}\right|\left(\left|\hat{\psi}-\Pi_{0} \hat{\psi}\right|\right)\left(|\hat{\psi}|+\left|\Pi_{0} \hat{\psi}\right|\right) d \hat{\sigma} d \hat{\tau} \\
& \quad \leq\left\|\hat{\psi}-\Pi_{0} \hat{\psi}\right\|\left(\int \hat{\tau}^{2}\left(-\eta_{0}+\frac{\hat{\tau}^{2}}{2}\right)^{4}\left(|\hat{\psi}|+\left|\Pi_{0} \hat{\psi}\right|\right)^{2} d \hat{\sigma} d \hat{\tau}\right)^{1 / 2} \\
& \quad \leq \sqrt{2}\left\|\hat{\psi}-\Pi_{0} \hat{\psi}\right\|\left(\int \hat{\tau}^{2}\left(-\eta_{0}+\frac{\hat{\tau}^{2}}{2}\right)^{4}\left(|\hat{\psi}|^{2}+\left|\Pi_{0} \hat{\psi}\right|^{2}\right) d \hat{\sigma} d \hat{\tau}\right)^{1 / 2} .
\end{aligned}
$$

We infer

$$
\begin{aligned}
& \left(\int \hat{\tau}^{2}\left(-\eta_{0}+\frac{\hat{\tau}^{2}}{2}\right)^{4}\left(|\hat{\psi}|^{2}+\left|\Pi_{0} \hat{\psi}\right|^{2}\right) d \hat{\sigma} d \hat{\tau}\right)^{1 / 2} \\
& \leq\left(\int \hat{\tau}^{2}\left(-\eta_{0}+\frac{\hat{\tau}^{2}}{2}\right)^{4}|\hat{\psi}|^{2} d \hat{\sigma} d \hat{\tau}\right)^{1 / 2} \\
& \quad+\left(\int \hat{\tau}^{2}\left(-\eta_{0}+\frac{\hat{\tau}^{2}}{2}\right)^{4}\left|\Pi_{0} \hat{\psi}\right|^{2} d \hat{\sigma} d \hat{\tau}\right)^{1 / 2} .
\end{aligned}
$$

Using the fact that $u_{\eta_{0}}$ is in the Schwartz class, we get

$$
\int \hat{\tau}^{2}\left(-\eta_{0}+\frac{\hat{\tau}^{2}}{2}\right)^{4}\left|\Pi_{0} \hat{\psi}\right|^{2} d \hat{\sigma} d \hat{\tau} \leq C \int\left\langle\hat{\psi}, u_{\eta_{0}}\right\rangle_{\hat{\tau}}^{2} d \hat{\sigma} \leq C\|\hat{\psi}\|^{2} .
$$


With the estimates of Agmon, we get

$$
\int \hat{\tau}^{2}\left(-\eta_{0}+\frac{\hat{\tau}^{2}}{2}\right)^{4}\left(|\hat{\psi}|^{2}+\left|\Pi_{0} \hat{\psi}\right|^{2}\right) d \hat{\sigma} d \hat{\tau} \leq C\|\hat{\psi}\|^{2}
$$

We deduce

$$
\left|\int \hat{\tau}\left(-\eta_{0}+\frac{\hat{\tau}^{2}}{2}\right)^{2}\left(|\hat{\psi}|^{2}-\left|\Pi_{0} \hat{\psi}\right|^{2}\right) d \hat{\sigma} d \hat{\tau}\right| \leq C h^{1 / 12}\|\hat{\psi}\|^{2} .
$$

In the same way, we get

$$
\left|\int\left(-\eta_{0}+\frac{\hat{\tau}^{2}}{2}\right) \hat{\tau}^{3}\left(|\hat{\psi}|^{2}-\left|\Pi_{0} \hat{\psi}\right|^{2}\right) d \hat{\sigma} d \hat{\tau}\right| \leq C h^{1 / 12}\|\hat{\psi}\|^{2} .
$$

Then, we can write

$$
\begin{aligned}
\int & \hat{\tau}\left(-\eta_{0}+\frac{\hat{\tau}^{2}}{2}\right)^{2}\left|\Pi_{0} \hat{\psi}\right|^{2} d \hat{\sigma} d \hat{\tau} \\
& =\left(\int \hat{\tau}\left(-\eta_{0}+\frac{\hat{\tau}^{2}}{2}\right)^{2}\left|u_{\eta_{0}}\right|^{2} d \hat{\tau}\right)\left(\int\left\langle\hat{\psi}, u_{\eta_{0}}\right\rangle_{\hat{\tau}}^{2} d \hat{\sigma}\right) .
\end{aligned}
$$

We get

$$
\int\left\langle\hat{\psi}, u_{\eta_{0}}\right\rangle_{\hat{\tau}}^{2} d \hat{\sigma}=\iint u_{\eta_{0}}^{2}\left\langle\hat{\psi}, u_{\eta_{0}}\right\rangle_{\hat{\tau}}^{2} d \hat{\sigma} d \hat{\tau}=\left\|\Pi_{0} \hat{\psi}\right\|^{2}=(1+o(1))\|\hat{\psi}\|^{2}
$$

We infer

$$
\begin{aligned}
& \int \hat{\tau}\left(-\eta_{0}+\frac{\hat{\tau}^{2}}{2}\right)^{2}\left|\Pi_{0} \hat{\psi}\right|^{2} d \hat{\sigma} d \hat{\tau} \\
& =\left(\int \hat{\tau}\left(-\eta_{0}+\frac{\hat{\tau}^{2}}{2}\right)^{2}\left|u_{\eta_{0}}\right|^{2} d \hat{\tau}\right)(1+o(1))\|\hat{\psi}\|^{2}
\end{aligned}
$$

In the same way, we get

$$
\begin{aligned}
& \int \hat{\tau}^{3}\left(-\eta_{0}+\frac{\hat{\tau}^{2}}{2}\right)\left|\Pi_{0} \hat{\psi}\right|^{2} d \hat{\sigma} d \hat{\tau} \\
& =\left(\int \hat{\tau}^{3}\left(-\eta_{0}+\frac{\hat{\tau}^{2}}{2}\right)\left|u_{\eta_{0}}\right|^{2} d \hat{\tau}\right)(1+o(1))\|\hat{\psi}\|^{2} .
\end{aligned}
$$

Gathering (4.4), (4.5), (4.6), (4.7), and (4.8) and the definition of $C_{0}$ in (1.10), we get the lower bound.

After rescaling, we deduce the following corollary. 
Corollary 4.4. There exist $h_{0}>0, C>0$ such that, for $h \in\left(0, h_{0}\right)$ and $\breve{\psi} \in \mathfrak{E}_{N}(h)$,

$$
\begin{aligned}
\check{Q}(\check{\psi}) \geq & \int \delta_{0}^{2 / 3}\left(\left|h D_{\tau} \check{\psi}\right|^{2}+\left|\left(\delta^{-1 / 6} i h \partial_{\sigma} \delta^{-1 / 6}-\eta_{0} h^{2 / 3}+\frac{\tau^{2}}{2}\right) \check{\psi}\right|^{2}\right) d \sigma d \tau \\
& +\frac{2}{3} \delta_{0}^{2 / 3} \alpha \nu_{1}\left(\eta_{0}\right) h^{4 / 3}\|\sigma \check{\psi}\|^{2}+C_{0} h^{5 / 3}\|\check{\psi}\|^{2}+o\left(h^{5 / 3}\right)\|\check{\psi}\|^{2} .
\end{aligned}
$$

We use the weighted Fourier transform defined in (3.17) and we infer the following result.

Corollary 4.5. There exist $h_{0}>0, C>0$ such that, for $h \in\left(0, h_{0}\right)$ and $\breve{\psi} \in \mathfrak{E}_{N}(h)$,

$$
\begin{aligned}
\check{Q}(\check{\psi}) \geq \int & \delta_{0}^{2 / 3}\left(\left|h D_{\tau} \check{\varphi}\right|^{2}+\left|\left(-h \mu-\eta_{0} h^{2 / 3}+\frac{\tau^{2}}{2}\right) \check{\varphi}\right|^{2}\right) d \mu d \tau \\
& +\frac{2}{3} \delta_{0}^{2 / 3} \alpha \nu_{1}\left(\eta_{0}\right) h^{4 / 3}\left\|D_{\mu} \check{\varphi}\right\|^{2}+C_{0} h^{5 / 3}\|\check{\varphi}\|^{2}+o\left(h^{5 / 3}\right)\|\check{\varphi}\|^{2},
\end{aligned}
$$

with $\check{\varphi}=\mathscr{F}_{\delta} \check{\psi}$.

Conclusion: proof of Theorem 1.11. Let us introduce the operator

$$
\frac{2}{3} \delta_{0}^{2 / 3} \alpha \nu_{1}\left(\eta_{0}\right) h^{4 / 3} D_{\mu}^{2}+\delta_{0}^{2 / 3}\left(h^{2} D_{\tau}^{2}+\left(-h \mu-\eta_{0} h^{2 / 3}+\frac{\tau^{2}}{2}\right)^{2}\right)+C_{0} h^{5 / 3}
$$

on $L^{2}\left(\mathbb{R}^{2}, d \sigma d \tau\right)$. We denote by $\tilde{\lambda}_{n}(h)$ its $n$-th eigenvalue. From Corollary 4.5 and the min-max principle, we deduce

$$
\lambda_{n}(h) \geq \tilde{\lambda}_{n}(h)+o\left(h^{5 / 3}\right) .
$$

The Born-Oppenheimer approximation (see [7] and [31]) provides the following estimate for $\tilde{\lambda}_{n}(h)$ :

$$
\tilde{\lambda}_{n}(h)=\theta_{0}^{n} h^{4 / 3}+\theta_{2}^{n} h^{5 / 3}+o\left(h^{5 / 3}\right) .
$$

The estimates (4.10) and (4.11) provide the conclusion. Let us recall the spirit of the Born-Oppenheimer approximation (see the historical reference [5]) without going into the details. The principle consists of replacing, for fixed $\mu$, the operator

$$
h^{2} D_{\tau}^{2}+\left(-h \mu-\eta_{0} h^{2 / 3}+\frac{\tau^{2}}{2}\right)^{2}
$$

by its lowest eigenvalue $h^{4 / 3} v_{1}\left(\eta_{0}+\mu h^{1 / 3}\right)$ and to analyze the spectrum of the "Born-Oppenheimer approximation" defined by

$$
h^{4 / 3} \delta_{0}^{2 / 3}\left(\frac{2}{3} \alpha v_{1}\left(\eta_{0}\right) D_{\mu}^{2}+v_{1}\left(\eta_{0}+\mu h^{1 / 3}\right)\right)
$$


This (semiclassical) analysis can be done through standard techniques (see [24], [25], and [15]). We can also refer two our recent works [4] and [37] where this idea appears. It can be proved (through Agmon estimates with respect to $\mu$ and a Feshbach-Grushin type argument) that the investigation reduces to the harmonic oscillator

$$
h^{4 / 3} \delta_{0}^{2 / 3}\left(\frac{2}{3} \alpha v_{1}\left(\eta_{0}\right) D_{\mu}^{2}+v_{1}\left(\eta_{0}\right)+h^{2 / 3} \frac{\nu_{1}^{\prime \prime}\left(\eta_{0}\right)}{2} \mu^{2}\right)
$$

and the estimate (4.11) follows.

\section{References}

[1] S. Agmon, Lectures on exponential decay of solutions of second-order elliptic equations: bounds on eigenfunctions of $N$-body Schrödinger operators. Mathematical Notes 29. Princeton University Press, Princeton, NJ, and University of Tokyo Press, Tokyo, 1982. MR 35B40 Zbl 0503.35001

[2] P. Bauman, D. Phillips, and Q. Tang, Stable nucleation for the Ginzburg-Landau system with an applied magnetic field. Arch. Rational Mech. Anal. 142 (1998), 1-43. MR 1629119 Zbl 0922.35157

[3] A. Bernoff and P. Sternberg, Onset of superconductivity in decreasing fields for general domains. J. Math. Phys. 39 (1998), 1272-1284. MR 1608449 Zbl 1056.82523

[4] V. Bonnaillie-Nöel, M. Dauge, N. Popoff, and N. Raymond, Discrete spectrum of a model Schrödinger operator on the half-plane with Neumann conditions. Z. Ange. Math. Phys. 63 (2011), 203-231. MR 2912345 Zbl 1238.35070

[5] M. Born and R. Oppenheimer, Zur Quantentheorie der Molekeln. Annalen d. Physik 84 (1927), 457-484. JFM 53.0845.04

[6] L. Charles and S. Vũ Ngọc, Spectral asymptotics via the semiclassical Birkhoff normal form. Duke Math. J. 143 (2008), 463-511. MR 2423760 Zbl 1154.58015

[7] J.-M. Combes, P. Duclos, and R. Seiler, The Born-Oppenheimer approximation. In G. Velo and A. Wightman (eds.), Rigorous atomic and molecular physics. Nato Science Series B 74. Springer Verlag, New York and London, 1981, 185-212.

[8] H. L. Cycon, R. G. Froese, W. Kirsch, and B. Simon, Schrödinger operators with application to quantum mechanics and global geometry. Texts and Monographs in Physics. Springer Verlag, Berlin etc., 1987. MR 883643 Zbl 0619.47005

[9] M. del Pino, P. L. Felmer, and P. Sternberg, Boundary concentration for eigenvalue problems related to the onset of superconductivity. Comm. Math. Phys. 210 (2000), 413-446. MR 1776839 Zbl 0982.35077

[10] N. Dombrowski and F. Germinet, and G. Raykov, Quantization of edge currents along magnetic barriers and magnetic guides. Ann. Henri Poincaré 12 (2011) 1169-1197. MR 2823212 Zbl 1230.81062

[11] S. Fournais and B. Helffer, Accurate eigenvalue asymptotics for the magnetic Neumann Laplacian. Ann. Inst. Fourier (Grenoble) 56 (2006) 0373-0956. MR 2228679 Zbl 1097.47020 
[12] S. Fournais and B. Helffer, Spectral methods in surface superconductivity. Progress in Nonlinear Differential Equations and their Applications 77. Birkhäuser Boston Inc., Boston, MA, 2010. MR 2662319 Zbl 1256.35001

[13] V. V. Grušin, Hypoelliptic differential equations and pseudodifferential operators with operator-valued symbols. Mat. Sb. (N.S.) 88 (1972) 504-521. English transl. Math. USSR, Sb. 17 (1973), 497-514. MR 0316879 Zbl 0255.35022

[14] M. Hara, A. Endo, Sh. Katsumoto, Y. Iye, Transport in two-dimensional electron gas narrow channel with a magnetic field gradient. Phys. Rev. B 69 (2004), Article Id. 153304.

[15] B. Helffer, Semi-classical analysis for the Schrödinger operator and applications. Lecture Notes in Mathematics 1336. Springer Verlag, Berlin etc., 1988. MR 960278 Zbl 0647.35002

[16] B. Helffer, Introduction to semi-classical methods for the Schrödinger operator with magnetic field. In A. El Soufi and M. Jazar (eds.), Aspects théoriques et appliqués de quelques EDP issues de la géométrie ou de la physique. Sémin. Congr. 17. Société Mathématique de France, Paris, 2009, 49-117. MR 2792093 Zbl 1225.81063

[17] B. Helffer, The Montgomery model revisited. Colloq. Math. 118 (2010), 391-400. MR 35P15 Zbl 1207.34110

[18] B. Helffer and Yu. A. Kordyukov, Spectral gaps for periodic Schrödinger operators with hypersurface magnetic wells: analysis near the bottom. J. Funct. Anal. 257 (2009), 3043-3081. MR 2568685 Zbl 1184.35233

[19] B. Helffer and Yu. A. Kordyukov, Semiclassical spectral asymptotics for a twodimensional magnetic Schrödinger operator: the case of discrete wells. In M. Braverman, L. Friedlander, T. Kappeler, P. Kuchment, P. Topalov, and J. Weitsman (eds.), Spectral theory and geometric analysis. International conference in honor of Mikhail Shubin's $65^{\text {th }}$ birthday, Northeastern University, Boston, MA, USA, July 29-August 2, 2009. Contemporary Mathematics 535. Amer. Math. Soc., Providence, RI, 2011, 55-78. MR 2560751 Zbl 1218.58017

[20] B. Helffer and A. Mohamed, Semiclassical analysis for the ground state energy of a Schrödinger operator with magnetic wells. J. Funct. Anal. 138 (1996), 40-81. MR 1391630 Zbl 0851.58046

[21] B. Helffer and A. Morame, Magnetic bottles in connection with superconductivity. J. Funct. Anal. 185 (2001), 604-680. MR 1856278 Zbl 1078.81023

[22] B. Helffer and A. Morame, Magnetic bottles for the Neumann problem: curvature effects in the case of dimension 3 (general case). Ann. Sci. École Norm. Sup. (4) 37 (2004), 105-170. MR 2050207 Zbl 1057.35061

[23] B. Helffer and M. Persson, Spectral properties of higher order anharmonic oscillators. J. Math. Sci. (N.Y.) 165 (2010), 110-126. MR 2838999

[24] B. Helffer and J. Sjöstrand, Multiple wells in the semiclassical limit I. Comm. Partial Differential Equations 9 (1984), 337-408. MR 740094

[25] B. Helffer and J. Sjöstrand, Puits multiples en limite semi-classique II. Interaction moléculaire. Symétries. Perturbation. Ann. Inst. H. Poincaré Phys. Théor. 42 (1985), 127-212. MR 798695 Zbl 0595.35031 
[26] M. A. Hoefer and M. I. Weinstein, Defect modes and homogenization of periodic Schrödinger operators. SIAM J. Math. Anal. 43 (2011) 971-996. MR 2801185 Zbl 1242.35032

[27] L. Hörmander, The analysis of linear partial differential operators III. Pseudo-differential operators. Reprint of the 1994 ed. Classics in Mathematics. Springer Verlag, Berlin, 2007. MR 2304165 Zbl 1115.35005

[28] T. Kato, Tosio Perturbation theory for linear operators. Die Grundlehren der mathematischen Wissenschaften 132. Springer Verlag, Berlin etc., 1966. MR 0203473 Zbl 0148.12601

[29] K. Lu and X.-B. Pan, Eigenvalue problems of Ginzburg-Landau operator in bounded domains. MR 1694223 Zbl 0943.35058

[30] K. Lu and X.-B. Pan, Surface nucleation of superconductivity in 3-dimensions. J. Differential Equations 168 (2000), 386-452. MR 1808455 Zbl 0972.35152

[31] A. Martinez, Développements asymptotiques et effet tunnel dans l'approximation de Born-Oppenheimer. Ann. Inst. H. Poincaré Phys. Théor. 50 (1989), 239-257. MR 1017965 Zbl 0689.35063

[32] R. Montgomery, Hearing the zero locus of a magnetic field. Comm. Math. Phys. 168 (1995), 651-675. MR 1328258 Zbl 0827.58076

[33] X.-B. Pan and K.-H. Kwek, Schrödinger operators with non-degenerately vanishing magnetic fields in bounded domains. Trans. Amer. Math. Soc. 354 (2002), 4201-4227. MR 1926871 Zbl 1004.35110

[34] N. Raymond, Sharp asymptotics for the Neumann Laplacian with variable magnetic field: case of dimension 2. Ann. Henri Poincaré 10 (2009), 95-122. MR 2496304 Zbl 1210.81034

[35] N. Raymond, On the semiclassical $3 D$ Neumann Laplacian with variable magnetic field. Asymptot. Anal. 68 (2010), 1-40. MR 2675335 Zbl 1200.35202

[36] N. Raymond, Semiclassical $3 D$ Neumann Laplacian with variable magnetic field: a toy model. Comm. Partial Differential Equations 37 (2012), 1528-1552. MR 2969489 Zbl 1254.35167

[37] N. Raymond, From the Laplacian with variable magnetic field to the electric Laplacian in the semiclassical limit. Preprint 2012. To appear.

hal.archives-ouvertes.fr/hal-00625260/

[38] J. Reijniers, A. Matulis, K. Chang, and F. M. Peeters, Quantum states in a magnetic anti-dot. Europhysics Letters 59 (2002), Article Id. 17013.

[39] S. Vũ Ngọc, Systèmes intégrables semi-classiques: du local au global. Panoramas et Synthèses 22. Société Mathématique de France, Paris, 2006. MR 2331010 Zbl 1118.37001

[40] S. Vũ Ngọc, Quantum Birkhoff normal forms and semiclassical analysis. In J.-P. Bourguignon, M. Kotani, Y. Maeda, and N. Tose (eds.), Noncommutativity and singularities. Proceedings of French-Japanese symposia held at IHÉS, Bures-sur-Yvette, France, November 20-23 and November 15-18, 2006. Mathematical Society of Japan, Tokyo, 2009, 99-116. MR 2463493 Zbl 1185.58012 
Received March 15, 2012; revised October 6, 2012

Nicolas Dombrowski, Laboratoire J. A. Dieudonné, UMR 7351, Université de Nice 06108 Nice Cedex 02, France

Home Page: www.nicolasdombrowski.com

E-mail: dombrowski@unice.fr

Nicolas Raymond, Laboratoire IRMAR, UMR 6625, Campus de Beaulieu, 35042 Rennes cedex, France

Home Page: perso.univ-rennes1.fr/nicolas.raymond/

E-mail: nicolas.raymond@univ-rennes1.fr 